\title{
Model-independent searches for new quarks at the LHC
}

\author{
Anupama Atre, ${ }^{a, b}$ Georges Azuelos, ${ }^{c, d}$ Marcela Carena, ${ }^{a, e}$ Tao Han, ${ }^{f}$ Erkcan Ozcan,,$^{g, h}$ \\ José Santiago ${ }^{i}$ and Gokhan Unel ${ }^{j}$ \\ ${ }^{a}$ Fermilab, Batavia, U.S.A. \\ ${ }^{b}$ Michigan State University, East Lansing, U.S.A. \\ ${ }^{c}$ University of Montreal, Montreal, Canada \\ ${ }^{d}$ TRIUMF, Vancouver, Canada \\ ${ }^{e}$ University of Chicago, Chicago, U.S.A. \\ ${ }^{f}$ University of Wisconsin, Madison, U.S.A. \\ ${ }^{g}$ University College, London, U.K. \\ ${ }^{h}$ Bogazici University, Istanbul, Turkey \\ ${ }^{i}$ University of Granada, Granada, Spain \\ ${ }^{j}$ University of California, Irvine, U.S.A. \\ E-mail: avatre@fnal.gov, georges.azuelos@umontreal.ca, \\ carena@fnal.gov, than@hep.wisc.edu, eo@hep.ucl.ac.uk, \\ jsantiago@ugr.es, Gokhan.Unel@cern.ch
}

ABSTRACT: New vector-like quarks can have sizable couplings to first generation quarks without conflicting with current experimental constraints. The coupling with valence quarks and unique kinematics make single production the optimal discovery process. We perform a model-independent analysis of the discovery reach at the Large Hadron Collider for new vector-like quarks considering single production and subsequent decays via electroweak interactions. An early LHC run with $7 \mathrm{TeV}$ center of mass energy and $1 \mathrm{fb}^{-1}$ of integrated luminosity can probe heavy quark masses up to $1 \mathrm{TeV}$ and can be competitive with the Tevatron reach of $10 \mathrm{fb}^{-1}$. The LHC with $14 \mathrm{TeV}$ center of mass energy and $100 \mathrm{fb}^{-1}$ of integrated luminosity can probe heavy quark masses up to $3.7 \mathrm{TeV}$ for order one couplings.

Keywords: Beyond Standard Model, Phenomenological Models, Hadronic Colliders

ARXiv EPRINT: 1102.1987 


\section{Contents}

1 Introduction $\quad 2$

2 General parameterization 3

2.1 Generic constraints on the couplings 3

2.2 Model considerations 4

2.2.1 Model I: two degenerate vector-like quark doublets 4

2.2.2 Model II: one vector-like quark doublet 6

$\begin{array}{ll}2.2 .3 \text { Model III: non-degenerate quarks } & 6\end{array}$

$\begin{array}{lll}2.3 & \text { Generic parameterization } & 7\end{array}$

$\begin{array}{lll}3 & \text { Heavy quark production } & 7\end{array}$

4 Heavy quark decay 11

5 Observability of the heavy quark signal $\quad \mathbf{1 2}$

5.1 Neutral current channel with $Z$-decays to charged leptons $\quad 16$

$\begin{array}{ll}5.2 & \text { Neutral current channel with } Z \text {-decays to neutrinos } \\ \end{array}$

$\begin{array}{ll}5.3 \text { Charged current channel } & 19\end{array}$

5.4 Identification of the heavy quark 23

6 Results 24

6.1 Current constraints from direct searches 24

6.2 Sensitivity at the LHC 25

6.3 Constraints on specific models 28
28.128

$\begin{array}{lll}\text { 6.3.1 } D \text { type singlet } & 28\end{array}$

$\begin{array}{lll}6.3 .2 & U \text { type singlet } & 30\end{array}$

6.3.3 Doublet with hypercharge 1/6 31

6.3.4 Degenerate bidoublet with hypercharge $1 / 6$ and $7 / 6 \quad 31$

6.4 Systematic effects 32

$\begin{array}{lll}7 & \text { Summary } & 32\end{array}$

$\begin{array}{ll}\text { A Explicit calculation of S } & 33\end{array}$

B Heavy fermion decay widths 


\section{Introduction}

The Large Hadron Collider (LHC) has finally started its quest to find the origin of electroweak symmetry breaking (EWSB). A natural realization of EWSB is likely to bring along new particles to cancel the ultraviolet sensitivity of the Higgs mass or to unitarize longitudinal gauge boson scattering in the absence of a light Higgs boson. A common occurrence among these new particles is new vector-like fermions that mix with Standard Model (SM) particles. Traditionally, vector-like quarks are considered to mix significantly only with the top sector, as the usual lore states that large mixings with lighter generations are excluded by electroweak precision or flavor observables. This statement is however not necessarily correct, as cancellations among the effects of different types of new quarks can significantly alleviate the indirect constraints [1-3]. It was recently pointed out [4] that these cancellations can be the result of a symmetry [5] and naturally arise in certain models of extra dimensions or strong EWSB, which also allow for relatively light 3rd generation partners [6-13]. Motivated by this type of models, several studies of the LHC reach for top partners [14-18], bottom partners [19, 20] and tau partners [21] have appeared recently in the literature. There are also some studies of new quarks mixing with first generation quarks. The discovery prospects of heavy down type vector-like quarks decaying to light quark generations in the context of the $E_{6}$ GUT model $[22,23]$ were studied in refs. [24, 25] for pair production mode and in ref. [26] for the single production mode. Most recently a more general analysis of the Tevatron reach for general new quarks with sizable couplings to the up or down quark was performed in ref. [4]. The goal of this article is to investigate the LHC potential in the search for general new heavy quarks exploring similar channels as in the previous study for the Tevatron.

Heavy quarks can be produced in pairs via strong QCD interactions

$$
q \bar{q}, g g \rightarrow Q \bar{Q},
$$

where $Q$ generically denotes a new heavy quark. This is the most common and modelindependent production mechanism for any states with QCD interactions. The heavy quarks can also be produced singly in flavor-changing processes via electroweak interactions

$$
q q^{\prime} \stackrel{V^{*}}{\longrightarrow} q_{1} Q,
$$

where $V=W$ or $Z$ gauge boson. Some of the current authors showed in ref. [4] that the Tevatron reach to find new vector-like quarks with sizable mixings to first generation SM quarks is significantly better in the single production channel than in pair production.

In this article we perform a theory-unbiased study of single production of new quarks with sizable mixing to the up or down quarks at the LHC. The large energy and luminosity of the LHC allow us to probe heavy new quark masses, even with rather moderate mixing to the first generation SM quarks, thus going beyond the need of exact cancellations to ensure compatibility with indirect constraints. We find quite encouraging results showing that an early run with $1 \mathrm{fb}^{-1}$ of integrated luminosity at a center of mass energy of $7 \mathrm{TeV}$ can be competitive with the Tevatron reach of $10 \mathrm{fb}^{-1}$. The mass reach with a larger integrated luminosity of $100 \mathrm{fb}^{-1}$ at a center of mass energy of $14 \mathrm{TeV}$ can be up to $3.7 \mathrm{TeV}$ for order one couplings. 
Amongst the many searches for new heavy quarks at collider experiments, one of the popular scenarios searched for is that of a sequential fourth generation quark [27-31]. Besides the limits on their masses from direct searches at the Tevatron there are significant, recently updated bounds from electroweak (EW) precision data [29-33]. The main difference between this model and the models under our consideration is that vector-like fermions, contrary to chiral fermions, decouple in the limit of large masses and therefore their effects can be made arbitrarily small, thus avoiding the severe constraints [34]. Phenomenologically, our theory parameterization and searching strategy are equally applicable to other heavy quark searches, as long as there is a sizable mixing with the light generations.

The rest of the paper is organized as follows. In section 2, we present a general model parameterization for the heavy quarks and discuss the constraints on various models. In section 3, we calculate the heavy quark production cross sections at the LHC for the single production processes. In section 4 , we calculate the heavy quark decay via the charged and neutral currents and parameterize them by a few model independent parameters. In section 5, we explore the observability of the heavy quarks at the LHC via the single production mechanism with the energy options for 7 and $14 \mathrm{TeV}$. We present a discussion of our results in section 6 and conclusions in section 7 .

\section{General parameterization}

In this section we present a general parameterization for models with new heavy quarks. Let us consider new quarks labeled by $Q=X, U, D, Y$ with electric charges

$$
Q_{X}=\frac{5}{3}, \quad Q_{U}=\frac{2}{3}, \quad Q_{D}=-\frac{1}{3}, \quad \text { and } \quad Q_{Y}=-\frac{4}{3},
$$

with masses $m_{Q}$ and arbitrary couplings to the SM gauge bosons and first generation quarks parameterized by

$$
\begin{aligned}
& \frac{g}{\sqrt{2}} W_{\mu}^{+}\left[\kappa_{u D} \bar{u}_{R} \gamma^{\mu} D_{R}+\kappa_{d Y} \bar{d}_{R} \gamma^{\mu} Y_{R}\right]+\frac{g}{\sqrt{2}} W_{\mu}^{-}\left[\kappa_{u X} \bar{u}_{R} \gamma^{\mu} X_{R}+\kappa_{d U} \bar{d}_{R} \gamma^{\mu} U_{R}\right] \\
& +\frac{g}{2 c_{W}} Z_{\mu}\left[\kappa_{u U} \bar{u}_{R} \gamma^{\mu} U_{R}+\kappa_{d D} \bar{d}_{R} \gamma^{\mu} D_{R}\right]+\text { h.c. }
\end{aligned}
$$

This choice of electric charges exhausts the possibilities of new quarks mixing through gauge couplings with SM quarks. For simplicity we have only considered RH couplings, which is not a restriction since we are not using angular correlations in our analysis. Possible Yukawa couplings between $U$ and $u$ or $D$ and $d$ have not been written explicitly since they will not be used in our analysis as discovery channels. Finally, we have included only one new quark of each charge as multiple new quarks for each charge can be added trivially.

\subsection{Generic constraints on the couplings}

The couplings in eq. (2.2) are subject to various constraints that can be classified as theoretical constraints and indirect experimental constraints. Theoretical constraints arise from the fact that $\kappa_{q Q}$ originates from the mixing of SM quarks with new vector-like quarks and 
it is therefore bound by the unitarity of the matrices involved in such mixing. Generically we have

$$
\kappa \leq 1
$$

although this constraint could be stronger in particular models (see below). Indirect experimental constraints are much more model-dependent. They can arise at tree level through modification of the SM fermion couplings or at one loop, mainly through contributions to the oblique parameters [35]. The former crucially depend on whether cancellations among the contributions due to the mixing with different heavy quarks are present or not. In the absence of cancellations, the couplings $\kappa_{q Q}$ can be quite constrained from low-energy experimental data. There is a generic correlation between the new couplings and corrections to the couplings of SM fermions, namely

$$
\frac{\delta g_{\mathrm{SM}}}{g_{\mathrm{SM}}} \sim \kappa^{2}
$$

where $g_{\mathrm{SM}}$ represents a generic SM coupling (see for instance ref. [36]). From EW precision observables, the SM couplings have been measured with a per mille precision. So, as a rule of thumb, we can expect a generic bound

$$
\kappa \lesssim \text { few } \times 10^{-2} \quad \text { (no cancellations) }
$$

As we will see below, cancellations among different contributions can however occur and make this bound completely disappear. One loop contributions to the oblique parameters, although also model dependent, are somewhat more robust. The resulting bounds on $\kappa_{q Q}$, which are typically milder than those in eq. (2.4), can nevertheless be the main constraint in some models.

\subsection{Model considerations}

As we have emphasized in the previous section, tree level experimental constraints on the values of $\kappa_{q Q}$ are very model dependent and can be, for a given theoretical model, a lot milder than that of eq. (2.4). The reason is that there are models in which some of the $\kappa_{q Q}$ can be sizable without modifying in an observable way the SM quark couplings (which are experimentally measured) and without inducing any observable flavor violation. This is the result of cancellations in the SM couplings due to the mixing of SM quarks with several different new quarks. These constraints or lack thereof can be better understood with a couple of illustrative examples as follows.

\subsubsection{Model I: two degenerate vector-like quark doublets}

The first model, discussed in detail in ref. [4], consists of two new vector-like quark electroweak doublets, with hypercharges $1 / 6$ and $7 / 6$ respectively, that mix only with the up quark, in the basis of diagonal Yukawa couplings for the charge 2/3 SM quarks. The particle content of these two doublets is two charge $2 / 3$, one charge $-1 / 3$ and one charge $5 / 3$ quark. If the two doublets are degenerate, i.e. they have the same mass and Yukawa couplings to $u$ before EWSB, their mixing with the up quark does not induce any observable correction to the SM quark couplings. This is due to the exact cancellation of two 


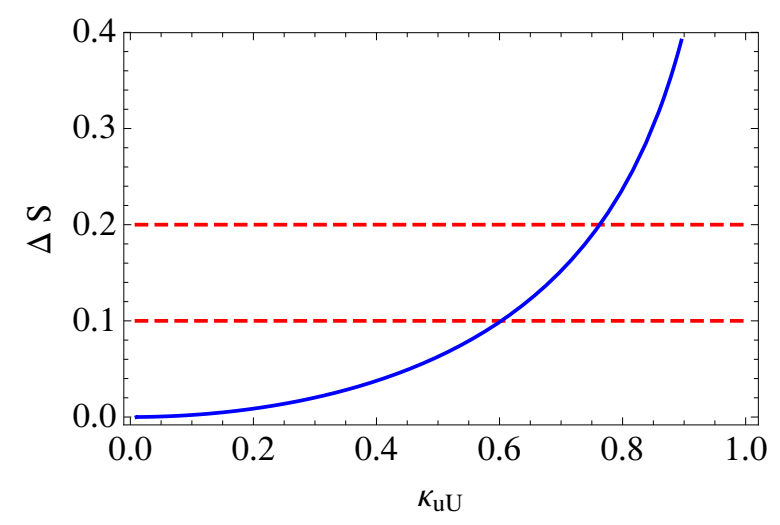

Figure 1. One loop contribution to the $S$ parameter, in the model with two degenerate doublets of hypercharge $1 / 6$ and $7 / 6$ and mixing only with the up quark, as a function of $\kappa_{u U}$. The result is independent of the mass of the heavy quarks up to corrections $\mathcal{O}\left(m_{u}^{2} / M^{2}\right)$.

large contributions (arising from the mixing of the up quark with the charge $2 / 3$ quarks in each doublet) with opposite signs. These large contributions are functions of $\kappa_{q Q}$ and since the large contributions cancel, we can have large values of $\kappa_{q Q}$ without upsetting any SM quark couplings. The only source of flavour violation in this case turns out to be suppressed by Cabbibo-Kobayashi-Maskawa (CKM) angles and by the ratio of the mass of the up quark and the mass of the new quarks, which provides enough suppression to keep flavor violation below experimental limits (details can be found in the appendix of ref. [4]). At the loop level, the new quarks do however contribute to oblique parameters. In the degenerate case, the new sector is custodially invariant and the $T$ parameter receives no correction. Interestingly enough, the contribution to the $S$ parameter turns out to be essentially independent of the mass of the heavy quarks, for fixed values of $\kappa_{u U}$. Note that for a fixed Yukawa coupling between the new quarks and the up quark, $\kappa_{u U}$ goes to zero in the large mass limit and therefore the vector-like quarks decouple in the heavy limit as they should. The dependence on the coupling $\kappa_{u U}$ is displayed in figure 1. Strict constraints based on the contribution to the oblique parameters are difficult to impose without knowledge of other possible sectors of the theory that could also contribute to them. Nevertheless, assuming $S \leq 0.2(0.1)$ gives a pretty mild bound of $\kappa_{u U} \lesssim 0.75(0.6)$ in the model under consideration. More details about the calculation of the $S$ parameter can be found in appendix A.

After EWSB one combination of the charge 2/3 heavy quarks couples to the up quark only through Yukawa couplings whereas the remaining three, of types $U, D$ and $X$ in our notation, have the following values of $\kappa$

$$
\kappa_{u U}=\sqrt{2} \kappa_{u D}=\sqrt{2} \kappa_{u X}=s_{R},
$$

where $s_{R}$ is the sine of the corresponding mixing angle which depends on the particular values of the model parameters. Thus, we see that in this model, the generic bound from the unitarity of the mixing is saturated for $\kappa_{u U} \leq 1$ and is a bit more stringent for $\kappa_{u D}=\kappa_{u X} \leq 1 / \sqrt{2}$. We refer the reader to ref. [4] for more details. 
Another similar model has two degenerate doublets of hypercharges $1 / 6$ and $-5 / 6$, respectively, where the heavy quarks mix only with the down quark. In this case there would be one combination of charge $-1 / 3$ heavy quarks that couples to the down quark only through Yukawa couplings, while the other three quarks, $U, D$ and $Y$ in our notation, have large couplings to $d_{R}$ with the following constraints

$$
\kappa_{d D}=\sqrt{2} \kappa_{d U}=\sqrt{2} \kappa_{d Y}=s_{R}
$$

resulting in a theory upper bound of $\kappa_{d D} \leq 1, \kappa_{d U}=\kappa_{d Y} \leq 1 / \sqrt{2}$. As we have stressed above, these cancellations can be due to a symmetry and therefore be natural.

\subsubsection{Model II: one vector-like quark doublet}

In our second example no cancellations occur and SM couplings are modified due to the mixing with the heavy quarks. Stringent experimental constraints arise on the mixing and therefore on the values of $\kappa_{q Q}$ (see eqs. (2.3)-(2.4)). The model consists of just one new electroweak doublet with hypercharge $1 / 6$ that mixes only with the up quark. The particle content is one $U$ and one $D$ quark and the mixing with the up quark induces an anomalous $Z u_{R} \bar{u}_{R}$ coupling, proportional to $\kappa_{u D}^{2}$, which is strongly constrained experimentally. A detailed analysis, using an updated version of the code in refs. [37, 38] gives a bound of $\kappa_{u D} \lesssim 0.07 .{ }^{1}$ The couplings to the heavy quarks can be computed as discussed in ref. [34] and part 13 of ref. [39] (see ref. [40] for explicit expressions). Denoting the sine and cosine of the mixing angle by $s_{R}$ and $c_{R}$, respectively, we have

$$
\kappa_{u U}=s_{R} c_{R}, \quad \kappa_{u D}=s_{R} .
$$

The indirect bound on $\kappa_{u D}$, from the constraint on the $Z u_{R} \bar{u}_{R}$ coupling, is therefore much more restrictive than the one coming from the unitarity of the mixing $\kappa_{u D} \leq 1$. It also induces a very restrictive constraint on $\kappa_{u U} \lesssim 0.07$. Incidentally, there are also loop contributions in this model to the $S$ and $T$ parameters but the tree level constraints are much more restrictive.

\subsubsection{Model III: non-degenerate quarks}

An intermediate model between our two examples, with two new doublets which are not exactly degenerate allows for partial cancellations. For instance, using the code in refs. [37, 38 ] we find that $\kappa_{u U} \sim 0.25$ is possible if it is within $\sim 10 \%$ of $\sqrt{2} \kappa_{u X}$. Assuming similar values of the Yukawa couplings, this can translate to a $5-15 \%$ difference between the masses of different quarks, which might be experimentally distinguishable.

Thus we can see that a large number of different possibilities can arise depending on the model but still sizable values of $\kappa_{q Q}$ cannot be excluded on a general basis.

\footnotetext{
${ }^{1}$ The code in refs. $[37,38]$ assumes symmetry between the first two generations. The reported bound therefore applies to a model with two new vector-like multiplets that mix equally with the first two generations. This can be considered a conservative but indicative bound in our model.
} 


\subsection{Generic parameterization}

In order to consider all these possibilities, we do a model-independent study, imposing no $a$ priori constraints on the values of $\kappa_{q Q}$. This way our results can be applied to any specific model, for which the relevant theoretical or experimental constraints will have to be taken into account.

We can parameterize the coupling $\kappa_{q Q}$ in a model-independent manner as

$$
\kappa_{q Q}=\left(v / m_{Q}\right) \tilde{\kappa}_{q Q},
$$

where the dimensionless parameter $\tilde{\kappa}_{q Q}$ encodes the model-dependence and $v \approx 174 \mathrm{GeV}$ is the Higgs vacuum expectation value. This parameterization is useful because we generically have

$$
\kappa_{q Q}=\frac{\lambda v}{m_{Q}}\left(1+\mathrm{O}\left(v^{2} / m_{Q}^{2}\right)\right)
$$

where $\lambda$ is some Yukawa coupling between SM and heavy quarks. Thus, in the limit that $\kappa_{q Q}$ is relatively small so that the expansion can be well approximated by the first term, $\tilde{\kappa}_{q Q}$ has a direct relation to a model parameter. It corresponds to a Yukawa coupling and it is naturally of order unity in a generic weakly coupled theory, and could therefore be up to $4 \pi$ in strongly coupled theories. For larger values of $\kappa_{q Q}$ non-linear effects become important and the dependence of $\tilde{\kappa}_{q Q}$ on the model parameters becomes much more complicated. It should be noted that all the bounds we have obtained for the couplings, both the experimental ones and the ones coming from the unitarity of the mixing, apply to $\kappa$ and not $\tilde{\kappa}$. Therefore heavier quarks can have a substantially larger value of $\tilde{\kappa}$ compatible with EW precision observables and unitarity of the mixing.

\section{Heavy quark production}

In this article, we discuss the single production of heavy quarks via the process shown in eq. (1.2). In order to keep our study widely applicable, we have factored out the model dependent coupling $\tilde{\kappa}^{2}$, so that the cross section depends only on the corresponding Parton Distribution Functions (PDFs) and the kinematics (mass of the heavy quark produced). These model-independent cross sections will be denoted as $\sigma_{Q}^{\mathrm{CC}}$ and $\sigma_{Q}^{\mathrm{NC}}$ for single production of quark $Q$ via charged current (CC) and neutral current (NC) interactions respectively. In the rest of the analysis, we adopt the CTEQ6L1 PDFs [41] for the parton distribution functions.

The single production cross section as a function of the mass of the heavy quark is shown in figures 2-4 for various quark species at two different c.m. energies, 7 and $14 \mathrm{TeV}$. We show separately the production cross section for each quark (solid black curve) and anti-quark (dashed black curve) species through charged $\left(W^{*}\right)$ or neutral $\left(Z^{*}\right)$ currents for single production. All the curves for single production correspond to a factorization and renormalization scale $\mu_{F}=\mu_{R}=m_{W}\left(m_{Z}\right)$ for CC (NC) processes. The scale dependence of our LO calculation is represented by colored bands that correspond to a scale variation up to $\mu_{F}=\mu_{R}=m_{Q}$. For comparison, the QCD cross section for pair production (independent 

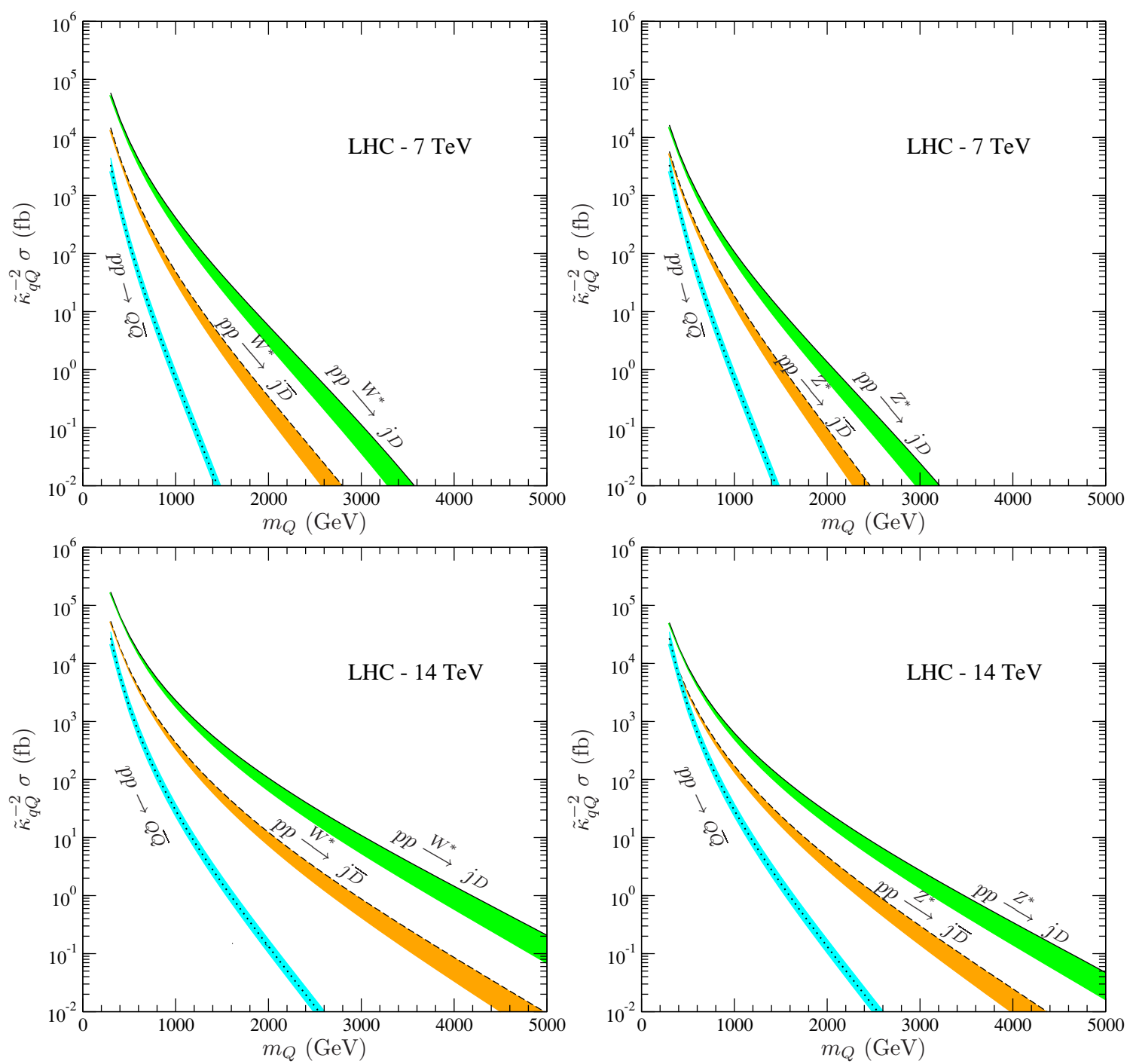

Figure 2. (a) Top left: cross sections (in fb) for singly producing the heavy quark $D$ via CC interactions versus its mass $m_{Q}$ at the LHC with $\sqrt{s}=7 \mathrm{TeV}$ in $p p$ collisions. (b) Top right: same as (a) but for NC interactions. (c) Bottom left: same as (a) but for $\sqrt{s}=14 \mathrm{TeV}$. (d) Bottom right: same as (b) but for $\sqrt{s}=14 \mathrm{TeV}$. Solid (dashed) curves represent production of $D(\bar{D})$ via the process $p p \rightarrow j D(\bar{D})$. The cross section for pair production (which is independent of $\tilde{\kappa}$ ) of heavy quark is shown as dotted (black) curve for comparison. The colored bands represent the variation in the leading order cross section due to the different scale choices as described in the text.

of $\tilde{\kappa}$ ) is also included in the figures and represented by dotted (black) curves. For pair production mode, we choose the central value of the factorization and renormalization scales to be $\mu_{F}=\mu_{R}=\sqrt{\hat{s}}$ and the scale dependence of our LO calculation is represented by colored bands that correspond to a scale variation up to $\sqrt{\hat{s}} / 2 \leq \mu_{F}=\mu_{R} \leq 2 \sqrt{\hat{s}}$.

Figure 2 and figure 3 correspond to single production of $D$ and $U$, respectively, with the left panels representing charged current channels and the right ones neutral current channels. Although we have included all contributing channels for the production, the 

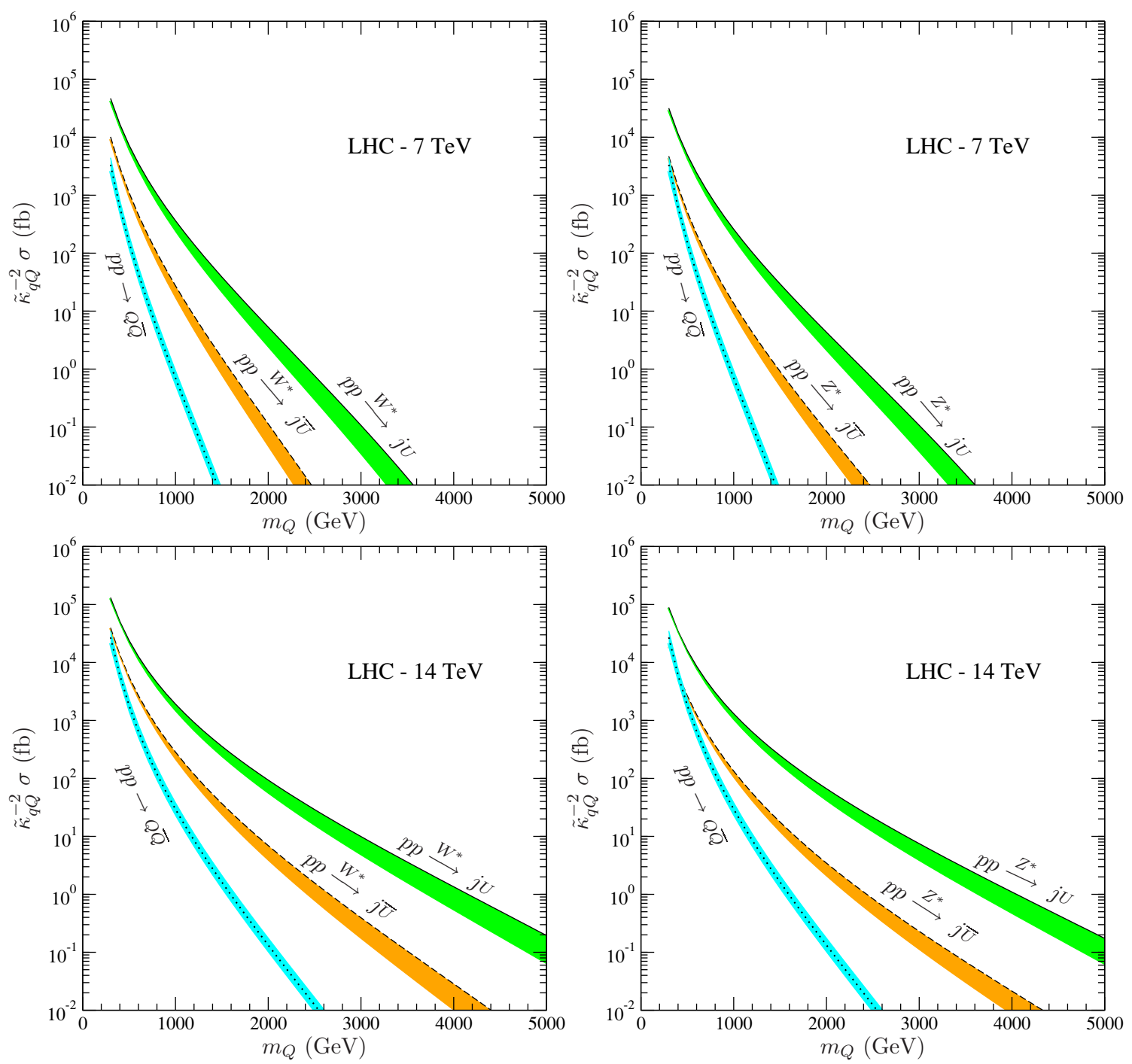

Figure 3. Same as figure 2 but for $U$.

leading contributions are from

$$
\begin{array}{ll}
d u \stackrel{W^{*}}{\rightarrow} j D, & q d \stackrel{Z^{*}}{\rightarrow} j D, \\
u d \stackrel{W^{*}}{\rightarrow} j U, & q u \stackrel{Z^{*}}{\rightarrow} j U,
\end{array}
$$

where $q$ denotes a generic valence quark parton and $j$ a light quark jet. Figure 4 on the other hand provides the production of $X$ on the left and $Y$ on the right. Due to their electric charge, they have only charged current channels and the leading contributions are from

$$
u u \stackrel{W^{*}}{\rightarrow} j X, \quad d d \stackrel{W^{*}}{\rightarrow} j Y .
$$

Several interesting features regarding single production at the LHC can be observed from figures 2-4. To start with, let us recall the advantages of single production versus pair production as shown in ref. [4]. The cross section for pair production falls off sharply due to phase space suppression and decreasing parton luminosity at large $x$ values. Evidently the 

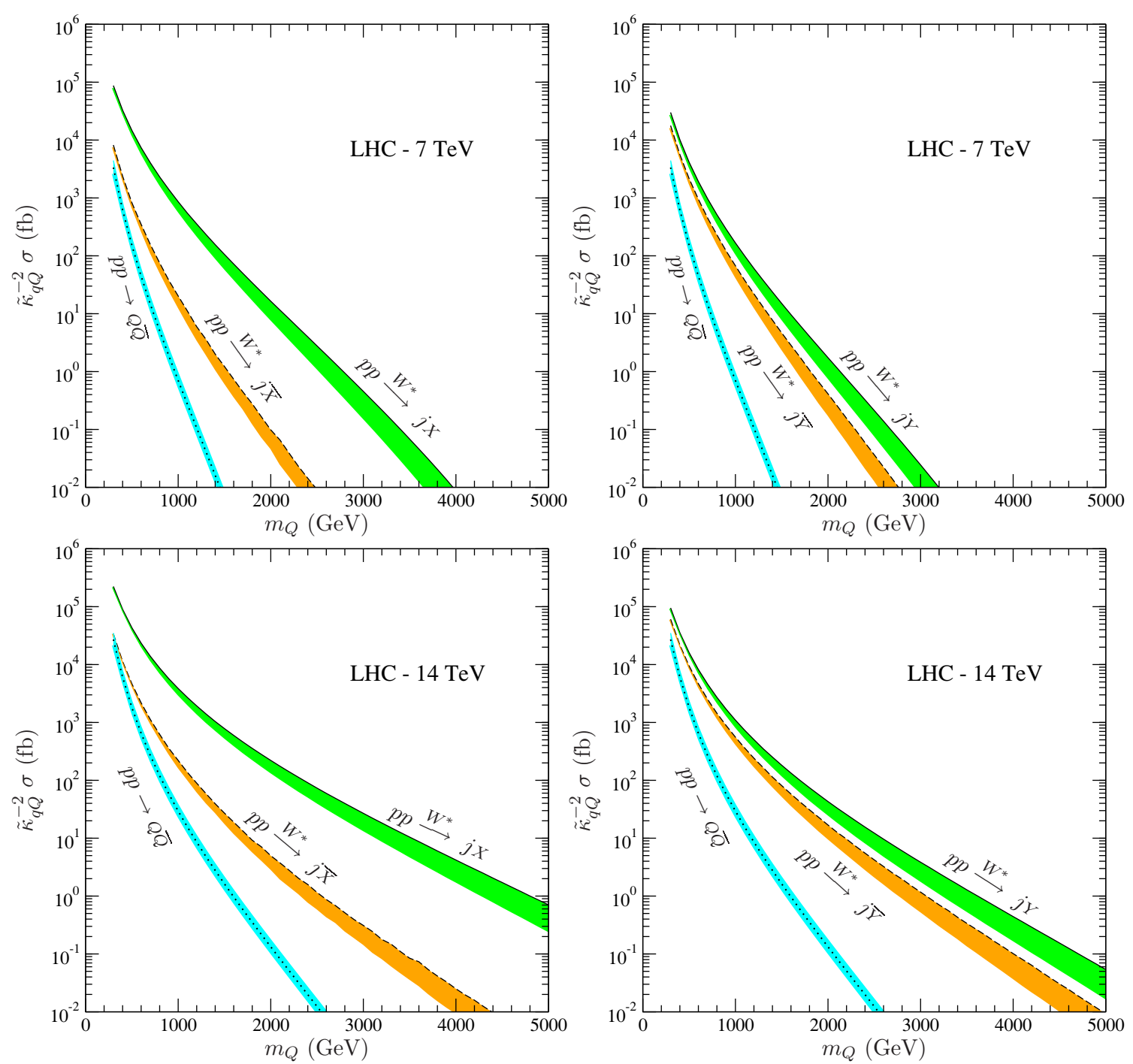

Figure 4. (a) Top left: cross sections (in fb) for singly producing the heavy quark $X$ via CC interactions versus its mass $m_{Q}$ at the LHC with $\sqrt{s}=7 \mathrm{TeV}$ in $p p$ collisions. (b) Top right: same as (a) but for the heavy quark Y. (c) Bottom left: same as (a) but for $\sqrt{s}=14 \mathrm{TeV}$. (d) Bottom right: same as (b) but for $\sqrt{s}=14 \mathrm{TeV}$. The description of the curves is the same as figure 2 but for $X$ and $Y$ with only charged current interactions.

LHC with its substantially larger c.m. energy will be able to probe higher quark masses in the pair production channel compared to the Tevatron. However, as at the Tevatron, single heavy quark production has the advantage of less phase-space suppression and longitudinal gauge boson enhancement of order $m_{Q}^{2} / M_{V}^{2}$ at higher energies compared to pair production. Due to the participation of $u, d$ valence quarks in the initial state with the coupling strength given in eq. (2.2), the cross section can be substantial and it falls more slowly for a higher mass. These effects can be easily observed from figures $2-4$. To summarize, the large c.m. energy of the LHC coupled with the above advantages of single production make this an ideal process for discovery of heavy quarks up to very large mass values and very small couplings. 


\begin{tabular}{|c|c|c|c|c|c|c|c|c|}
\hline $\begin{array}{c}\text { Channel } \\
\left(m_{Q}\right)\end{array}$ & $\begin{array}{c}7 \mathrm{TeV} \\
(0.9 \mathrm{TeV})\end{array}$ & $\begin{array}{c}14 \mathrm{TeV} \\
(1.8 \mathrm{TeV})\end{array}$ & Channel & $\begin{array}{c}7 \mathrm{TeV} \\
(0.9 \mathrm{TeV})\end{array}$ & $\begin{array}{c}14 \mathrm{TeV} \\
(1.8 \mathrm{TeV})\end{array}$ & $\begin{array}{c}\text { Channel } \\
7 \mathrm{TeV} \\
(0.9 \mathrm{TeV})\end{array}$ & $\begin{array}{c}14 \mathrm{TeV} \\
(1.8 \mathrm{TeV})\end{array}$ \\
\hline$p p \stackrel{W^{*}}{\longrightarrow} j D$ & 0.69 & 0.18 & $p p \stackrel{W^{*}}{\longrightarrow} j U$ & 0.61 & 0.16 & $p p \stackrel{W^{*}}{\longrightarrow} j X$ & 1.4 & 0.36 \\
$p p \stackrel{W^{*}}{\longrightarrow} j \bar{D}$ & 0.089 & 0.022 & $p p \stackrel{W^{*}}{\longrightarrow} j \bar{U}$ & 0.052 & 0.013 & $p p \stackrel{W^{*}}{\longrightarrow} j \bar{X}$ & 0.037 & 0.0092 \\
$p p \stackrel{Z^{*}}{\longrightarrow} j D$ & 0.18 & 0.047 & $p p \stackrel{Z^{*}}{\longrightarrow} j U$ & 0.43 & 0.11 & $p p \stackrel{W^{*}}{\longrightarrow} j Y$ & 0.29 & 0.074 \\
$p p \stackrel{Z^{*}}{\longrightarrow} j \bar{D}$ & 0.034 & 0.0088 & $p p \stackrel{Z^{*}}{\longrightarrow} j \bar{U}$ & 0.025 & 0.0064 & $p p \stackrel{W^{*}}{\longrightarrow} j \bar{Y}$ & 0.12 & 0.031 \\
\hline
\end{tabular}

Table 1. Cross sections in pb for EW single production of $D, U, X$ and $Y$ with $\tilde{\kappa}=1$ and $m_{Q}=900(1800) \mathrm{GeV}$ at the LHC with $\sqrt{s}=7$ (14) TeV.

Heavy quarks are produced at a much higher rate compared to anti-quarks with a larger difference for higher masses at the LHC. This difference in the rates of production of $Q$ and $\bar{Q}$ is due to the difference in the PDFs of valence and sea quarks in the initial states - heavy quarks couple to valence quarks whereas heavy anti-quarks couple to sea quarks. As we will see in later sections this feature will help us in constraining the electromagnetic charge of the heavy quarks. For $j Q$ production valence quarks participate in the initial state and as the quark PDFs dominate at higher energies, the cross section falls slowly. Also note that, the relative sizes of the single production cross sections between different types of quarks are determined by the corresponding valence quark density in the initial state. To illustrate the features of single production explicitly, the cross sections for EW single production computed with MadGraph/MadEvent [42] are listed in table 1 for all $Q$ with $m_{Q}=900$ (1800) $\mathrm{GeV}$ and $\tilde{\kappa}=1$ at the LHC with $\sqrt{s}=7$ (14) TeV. These results reiterate the previous observations that (i) valence quarks dominate; (ii) $u$ quark contributes more than $d$; and (iii) NC is weaker than CC.

\section{Heavy quark decay}

The singly produced heavy quarks will decay into jets and gauge or Higgs bosons through gauge and Yukawa interactions. In this article we are not considering the Higgs related channels so the relevant decay channels are

$$
\begin{array}{ll}
X \rightarrow W^{+} u, & Y \rightarrow W^{-} d, \\
U \rightarrow W^{+} d, Z u, & D \rightarrow W^{-} u, Z d,
\end{array}
$$

and the charge conjugated ones.

To perform a model-independent study, we classify processes according to their final states. In order to overcome the QCD background, we consider leptonic decays of the corresponding gauge boson. Therefore, the four final state configurations that could be experimentally distinguished from the SM backgrounds are:

$$
j j\left(W^{-} \rightarrow \ell^{-} \bar{\nu}\right), \quad j j\left(W^{+} \rightarrow \ell^{+} \nu\right), \quad j j\left(Z \rightarrow \ell^{+} \ell^{-}\right), \quad j j(Z \rightarrow \nu \bar{\nu}) .
$$

Following this classification, we parameterize the corresponding cross section, under the narrow width approximation as

$$
\sigma\left(p p \rightarrow j j f f^{\prime}\right)=S^{V} \operatorname{Br}\left(V \rightarrow f f^{\prime}\right)
$$


where $V=W^{+}, W^{-}$and $Z$ with $f f^{\prime}=\ell^{+} \nu, \ell^{-} \bar{\nu}$ and $\ell^{+} \ell^{-}$plus $\nu \bar{\nu}$, respectively.

All the model dependence is encoded in the $S^{V}$ parameters which are nothing but the production cross sections times the corresponding branching ratios summed over all possible channels. These can be written as:

$$
\begin{aligned}
S^{W^{-}} \equiv & {\left[\tilde{\kappa}_{d D}^{2} \sigma_{D}^{\mathrm{NC}}+\tilde{\kappa}_{u D}^{2} \sigma_{D}^{\mathrm{CC}}\right] \operatorname{Br}\left(D \rightarrow W^{-} u\right)+\tilde{\kappa}_{d Y}^{2} \sigma_{Y}^{\mathrm{CC}} \operatorname{Br}\left(Y \rightarrow W^{-} d\right) } \\
& +\left[\tilde{\kappa}_{u U}^{2} \sigma_{\bar{U}}^{\mathrm{NC}}+\tilde{\kappa}_{d U}^{2} \sigma_{\bar{U}}^{\mathrm{CC}}\right] \operatorname{Br}\left(\bar{U} \rightarrow W^{-} \bar{d}\right)+\tilde{\kappa}_{u X}^{2} \sigma_{\bar{X}}^{\mathrm{CC}} \operatorname{Br}\left(\bar{X} \rightarrow W^{-} \bar{u}\right), \\
S^{W^{+}} \equiv & {\left[\tilde{\kappa}_{d D}^{2} \sigma_{\bar{D}}^{\mathrm{NC}}+\tilde{\kappa}_{u D}^{2} \sigma \frac{\mathrm{CC}}{D}\right] \operatorname{Br}\left(\bar{D} \rightarrow W^{+} \bar{u}\right)+\tilde{\kappa}_{d Y}^{2} \sigma_{\bar{Y}}^{\mathrm{CC}} \operatorname{Br}\left(\bar{Y} \rightarrow W^{+} \bar{d}\right) } \\
& +\left[\tilde{\kappa}_{u U}^{2} \sigma_{U}^{\mathrm{NC}}+\tilde{\kappa}_{d U}^{2} \sigma_{U}^{\mathrm{CC}}\right] \operatorname{Br}\left(U \rightarrow W^{+} d\right)+\tilde{\kappa}_{u X}^{2} \sigma_{X}^{\mathrm{CC}} \operatorname{Br}\left(X \rightarrow W^{+} u\right), \\
S^{Z} \equiv & {\left[\tilde{\kappa}_{d D}^{2}\left(\sigma_{D}^{\mathrm{NC}}+\sigma \frac{\mathrm{NC}}{D}\right)+\tilde{\kappa}_{u D}^{2}\left(\sigma_{D}^{\mathrm{CC}}+\sigma \frac{\mathrm{CC}}{D}\right)\right] \operatorname{Br}(D \rightarrow Z d) } \\
& +\left[\tilde{\kappa}_{u U}^{2}\left(\sigma_{U}^{\mathrm{NC}}+\sigma_{\bar{U}}^{\mathrm{NC}}\right)+\tilde{\kappa}_{d U}^{2}\left(\sigma_{U}^{\mathrm{CC}}+\sigma_{\bar{U}}^{\mathrm{CC}}\right)\right] \operatorname{Br}(U \rightarrow Z u),
\end{aligned}
$$

where $\sigma_{Q}^{\mathrm{CC}, \mathrm{NC}}$ are defined in section 3 and displayed in figures $2-4$.

These $S^{V}$ parameters are what we are sensitive to from the experimental point of view. Their apparently complicated form is due to the high degree of model independence but can simplify greatly in specific models. For instance, assuming two degenerate doublets of hypercharges $1 / 6$ and $7 / 6$ that only mix with the up quark [4], we have three quarks of charges $5 / 3,2 / 3$ and $-1 / 3$, all with the same mass $m_{Q}$, each one decaying with $100 \%$ branching ratio to $W^{+} u, Z u$ and $W^{-} u$, respectively. The fourth quark with charge $2 / 3$, decays via the $H u$ channel and we do not consider that here. In that case we have

$$
S^{W^{-}}=\tilde{\kappa}_{u D}^{2} \sigma_{D}^{\mathrm{CC}}+\tilde{\kappa}_{u X}^{2} \sigma_{\bar{X}}^{\mathrm{CC}}, \quad S^{W^{+}}=\tilde{\kappa}_{u X}^{2} \sigma_{X}^{\mathrm{CC}}+\tilde{\kappa}_{u D}^{2} \sigma_{\bar{D}}^{\mathrm{CC}}, \quad S^{Z}=\tilde{\kappa}_{u U}^{2}\left(\sigma_{U}^{\mathrm{NC}}+\sigma_{\bar{U}}^{\mathrm{NC}}\right),
$$

with $\tilde{\kappa}_{u U}=\sqrt{2} \tilde{\kappa}_{u D}=\sqrt{2} \tilde{\kappa}_{u X} \leq m_{Q} / v$, where the inequality is the theoretical bound as discussed in section 2.2.1. In this specific case with two degenerate doublets it then implies the following upper bound on the corresponding $S^{V}$,

$$
\begin{aligned}
S^{W^{-}} & \leq \frac{1}{2}\left(\frac{m_{Q}}{v}\right)^{2}\left(\sigma_{D}^{\mathrm{CC}}+\sigma_{\bar{X}}^{\mathrm{CC}}\right), \quad S^{W^{+}} \leq \frac{1}{2}\left(\frac{m_{Q}}{v}\right)^{2}\left(\sigma_{X}^{\mathrm{CC}}+\sigma_{\bar{D}}^{\mathrm{CC}}\right), \\
S^{Z} & \leq\left(\frac{m_{Q}}{v}\right)^{2}\left(\sigma_{U}^{\mathrm{NC}}+\sigma_{\bar{U}}^{\mathrm{NC}}\right) .
\end{aligned}
$$

The expressions for other models can be easily computed using the general expressions for the partial widths given in appendix B. In the next section we describe the prospects for the observability of the new heavy quarks according to the signatures defined in this section.

\section{Observability of the heavy quark signal}

The classification of the possible final states that we have performed in the previous section allows us to perform a model-independent analysis of the LHC reach of new quarks which have sizable mixing with first generation quarks. We report on the results of such an analysis in the next few sections for single production. After a general discussion of the main kinematical features of the signal and background, we discuss the results of such 


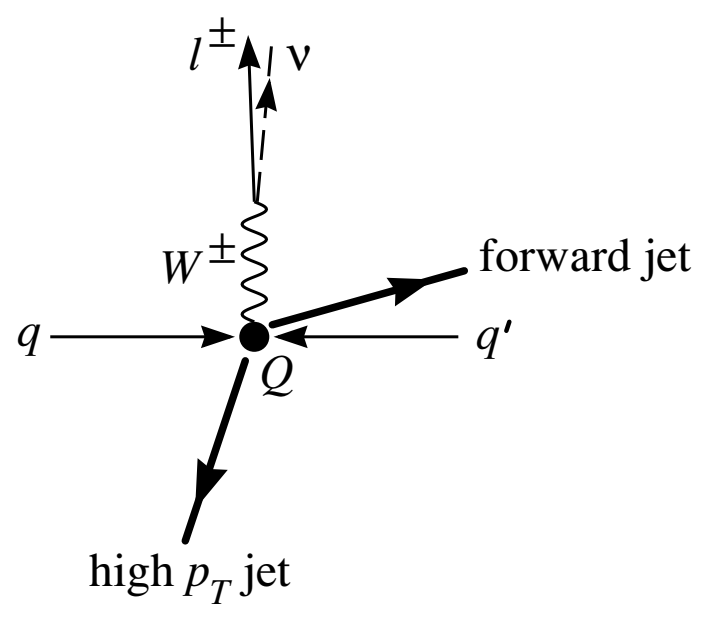

Figure 5. Illustration of the signal kinematics for a single heavy quark production.

an analysis for two different scenarios, one early run with $1 \mathrm{fb}^{-1}$ integrated luminosity at $\sqrt{s}=7 \mathrm{TeV}$ and one longer run with $100 \mathrm{fb}^{-1}$ of integrated luminosity at $\sqrt{s}=14 \mathrm{TeV}$.

The final state channels of our current interest are

$$
j Q \rightarrow \ell^{ \pm} \mathbb{F}_{T} 2 j, \quad \ell^{+} \ell^{-} 2 j, \quad \mathbb{F}_{T} 2 j,
$$

from $Q$ decaying to a light-quark jet plus a $W^{ \pm}\left(\rightarrow \ell^{ \pm} \nu\right)$ or $Z\left(\rightarrow \ell^{+} \ell^{-}, \nu \bar{\nu}\right)$, respectively. For the leptonic decay modes of the gauge bosons we consider $\ell=e, \mu$ and $\nu=\nu_{e}, \nu_{\mu}, \nu_{\tau}$. Although the inclusion of the $\tau$ lepton in the final state could increase the signal statistics, for simplicity we ignore this experimentally more challenging channel. The relevant backgrounds to these processes are

- $W+$ jets, $Z+$ jets with $W, Z$ leptonic decays;

- $W^{+} W^{-}, W^{ \pm} Z$ and $Z Z$ with semi-leptonic decays;

- single top production leading to $W^{ \pm} b q$;

- top pair production with fully leptonic and semi-leptonic decays.

An important aspect for our proposed search is the unique kinematics of single production and we discuss it first and present the detailed analysis next. A simple cartoon representation of the kinematic features is shown in figure 5. First, one of the two jets is from the heavy quark decay that makes it very energetic with a Jacobian peak in the transverse momentum spectrum near

$$
p_{T}(j) \approx \frac{1}{2} m_{Q}\left(1-M_{V}^{2} / m_{Q}^{2}\right)^{1 / 2}
$$

Using the $p_{T}$ of the jets as a discriminant gives very good accuracy in identifying the jet coming from the decay of the heavy quark correctly, especially for high masses. Hence we identify the hardest jet $\left(j_{1}\right)$ in the event as the one from heavy quark decay. The second jet 
is associated with the $t$-channel exchange of a gauge boson. The collinear behavior of the radiation implies a forward outgoing jet with its transverse momentum governed by the propagator's mass, typically around $M_{W} / 2$, and with a high rapidity. The pseudo-rapidity of the jet associated with the $t$-channel exchange of the gauge boson peaks at large values (typically $|\eta| \gtrsim 2$ ). The soft nature of the radiated gauge boson makes the accompanying jet energetic. These features can be used for forward jet tagging to enhance the signal over the backgrounds. The forward tagged jet candidate is selected as the one with the highest deposited total energy and a minimum threshold in the pseudo-rapidity separation between the tag jet candidate and the decay products of the heavy quark, namely the reconstructed gauge boson and the highest $p_{T}$ jet, in addition to the basic cuts for jets during generation. These selection criteria accurately identify the forward tagged jet $\left(j_{t}\right)$ as the one associated with the $t$-channel exchange of a gauge boson. Note that there is also an $s$-channel diagram where the jet is not necessarily forward but the contribution of this channel is negligible.

Similar to the hard jet, the $W / Z$ from the heavy quark decay is also very energetic. This leads to rather collimated final state leptons in the signal while those leptons in the background still tend to be back-to-back. The central hard jet and the energetic gauge boson $W / Z$ are both from the heavy quark decay and are thus nearly back-to-back in the transverse plane. The separation $(\Delta R)$ of the central hard jet and the tagged jet is typically large for the signal as well, while that for the QCD background tends to present a collinear singularity.

Of most importance is the reconstruction of the mass peak for the resonant particles. For a heavy quark decay with $Z \rightarrow \ell^{+} \ell^{-}$in the final state, it is straightforward to form the invariant mass of the heavy quark $m_{Q}=M\left(j_{1}, Z\right)$ for the signal and backgrounds. For the final state with $W \rightarrow \ell \nu$ and $Z \rightarrow \nu \bar{\nu}$, one can define a cluster transverse mass variable to be

$$
M_{T}^{2}=\left(\sqrt{p_{T W, Z}^{2}+M_{W, Z}^{2}}+p_{T j_{1}}\right)^{2}-\left(\vec{p}_{T W, Z}+\vec{p}_{T j_{1}}\right)^{2} .
$$

However, even for the $W \rightarrow \ell \nu$ channel a full invariant mass can be constructed by including the $W$ rest mass approximation (details are presented in section 5.3). Next, we present some details about the simulation.

We have performed a detailed simulation of both the signal and all the relevant backgrounds using MadGraph/MadEvent [42] for event generation at the partonic level and PYTHIA [43] for parton showering with initial and final state radiation, as well as hadronization. Fast detector simulation was performed using DeLPHES [44], version 1.8b, with the particle flow option turned off and the jets reconstructed using the anti- $K_{\mathrm{T}}$ algorithm with a radius of 0.7 and a minimum transverse momentum of $20 \mathrm{GeV}$. All other parameters were set to default values that correspond to the ATLAS Detector. Missing energy was manually corrected for the muons in the event.

The CTEQ6L1 PDFs [41] were used for both signal and background samples. For the signal we set the factorization and renormalization scales to be $\mu_{F}=\mu_{R}=m_{W}\left(m_{Z}\right)$ for CC (NC) processes. Due to the nature of the vector boson fusion, it is known that the natural choice of the factorization scale is around the mass of the propagating particle [45, 46]. Varying the scale within some reasonable range could result in an uncertainty of about 


\begin{tabular}{|c|c|c|}
\hline Background & $\sqrt{s}=7 \mathrm{TeV}$ & $\sqrt{s}=14 \mathrm{TeV}$ \\
\hline$(W \rightarrow \ell \nu)+1,2,3$ jets & 1070 & 2680 \\
\hline$(W \rightarrow \ell \nu)+2$ jets & & \\
$p_{T}\left(j_{1}\right)>400 \mathrm{GeV}$ & - & 2.76 \\
\hline$(Z \rightarrow \nu \nu)+1,2,3$ jets & 402 & 1206 \\
\hline$(Z \rightarrow \ell \ell)+1,2,3$ jets & 97.8 & 247 \\
\hline$W_{\ell \nu}^{ \pm} W_{j j}^{\mp}+0,1,2$ jets & 7.83 & 19.0 \\
\hline$W_{\ell \nu}^{ \pm} Z_{j j}+0,1$ jets & 1.64 & 4.18 \\
$W_{j j}^{ \pm} Z_{\ell \ell}+0,1$ jets & 0.429 & 1.05 \\
\hline$Z_{\ell \ell} Z_{j j}+0,1,2$ jets & 0.291 & 0.689 \\
\hline single top $\left(t \rightarrow \ell^{ \pm} \nu b\right)+1,2$ jets & 7.24 & 28.8 \\
\hline$t \bar{t}$ fully leptonic & 3.73 & 21.1 \\
\hline$t \bar{t}$ semi leptonic & 20.4 & 121 \\
\hline
\end{tabular}

Table 2. Cross section (in pb) for the various background processes with the acceptance cuts in eq. (5.3). A requirement that $p_{T}\left(j_{1}\right)>40 \mathrm{GeV}$ was applied in all cases, except where indicated.

10\%. For the background processes the renormalization and factorization scales are set to $\mu_{F}=\mu_{R}=Q^{2}=\sum\left(m^{2}+p_{T}^{2}(j)\right)$. To avoid double counting of the phase space overlap between the parton shower and matrix element parton generation, the modified MLM matching procedure [47] with the showerkt method [48] was applied for the background processes with multijets in the final state. The value of the parameter xqcut, which sets the minimum $K_{T}$ measure of partons in the matrix element generation, was set to $30 \mathrm{GeV}$. We summarize below the minimum cuts imposed in reconstructing the physics objects for our analysis.

$$
\begin{aligned}
& p_{T}\left(j_{1}\right)>40 \mathrm{GeV}, \quad p_{T}^{j}>20 \mathrm{GeV}, \quad p_{T}^{\ell}>15 \mathrm{GeV}, \\
& \left|\eta_{j}\right|<5, \quad\left|\eta_{\ell}\right|<2.5, \quad \Delta R(j \ell)>0.4, \quad \Delta R(\ell \ell)>0.2 .
\end{aligned}
$$

Various background cross sections are given in table 2. Finally QCD di-jet samples at various hard-scattering momenta $\left(\hat{p}_{\perp}\right)$ were generated with PYTHIA to be used as backgrounds to the $Z_{\nu \nu}+2$ jets analysis. The cross-section for the $280 \mathrm{GeV}<\hat{p}_{\perp}<560 \mathrm{GeV}$ range, which is found to be the most relevant in terms of mimicking the signal, is $1.82 \mathrm{nb}$ at $7 \mathrm{TeV}$ collision energy.

It is evident from table 2 that $W / Z+$ jets is the leading background. However there is also a sizable contribution to the total background from $t \bar{t}$ production. While the cuts we describe later are sufficient to extract the signal from the large backgrounds, we list below some further improvements that can be made in order to reduce the large $t \bar{t}$ background. For the case of CC decay modes of the signal, we can impose a veto on a second isolated lepton to reduce the $t \bar{t}$ background from the fully leptonic decay mode. For the NC decay mode of the signal with leptons in the final state, we can veto events with large missing energy. To further reduce $t \bar{t}$ events, we can veto events with $b$-tags (this has virtually no effect on our signal). The semi-leptonic decay mode can be reduced further by vetoing events where any two jets reconstruct a $W$-boson or if any three jets reconstruct the top 
quark. These simple vetoes, together with the rest of the cuts allow us to reduce the $t \bar{t}$ background to negligible levels. In the next three subsections we present the $\mathrm{CC}$ and $\mathrm{NC}$ analyses making use of the unique kinematic features discussed above.

For the rest of the analyses, we consider a variety of heavy quark masses and study the reach at two LHC energies, as given below

$$
\begin{array}{ll}
m_{Q}=300,600,900 \mathrm{GeV}, & \sqrt{s}=7 \mathrm{TeV} \\
\left.m_{Q}=900,1800,2400 \text { (or } 3000\right) \mathrm{GeV}, & \sqrt{s}=14 \mathrm{TeV} .
\end{array}
$$

\subsection{Neutral current channel with $Z$-decays to charged leptons}

In this section we study the case where the heavy quark decays into a jet and a $Z$ boson, with the $Z$ boson decaying leptonically into electrons and muons $\left(\ell^{ \pm}=e^{ \pm}, \mu^{ \pm}\right)$and investigate the prospects of the LHC in this channel. Since the $Z$-boson's invariant mass can most easily be reconstructed using the charged leptons, the analysis starts with the selection of those. The basic acceptance cuts are listed in eq. (5.3). The invariant mass reconstructed using the two leptons is required to be within $25 \mathrm{GeV}$ of the $Z$-boson mass peak to remove any possible fakes and misidentifications. Additionally, to select the boosted $Z$ bosons from the signal, the azimuthal angle between the two leptons is required to be smaller than a maximum, the value of which is optimized for the best signal significance.

The event is also required to contain at least two jets isolated from leptons and other jets, the criteria for which are listed in eq. (5.3). The jet with the highest $p_{T}$ is selected as the one coming from the heavy quark decay. The tag jet candidate is selected as the jet with the highest deposited energy and a minimum threshold on the pseudorapidity separation between the tag jet candidate and the decay products of the heavy quark, namely, the reconstructed $Z$ boson and the jet with the highest $p_{T}$. To take advantage of the forward nature of the tagged jet, thresholds optimized for best signal significance are applied for the pseudorapidity of the tagged jet. The efficiencies of these selection criteria in the order they are applied for various signal and background samples are shown in table 3 for the $7 \mathrm{TeV}$ and $14 \mathrm{TeV}$ c.m. energy scenarios.

The final heavy quark $(Q)$ candidate's invariant mass is formed using the reconstructed $\mathrm{Z}$ boson candidate and the jet with the highest transverse momentum. The results of this are shown in figure 6(a) for a heavy $U$ type quark with mass of $600 \mathrm{GeV}$ for $\sqrt{s}=$ $7 \mathrm{TeV}$. The black, blue and red histograms represent background, signal and signal plus background respectively. In order to extract the signal significance, a series of successive fits are performed to the signal+background sample. The procedure starts with a Landau functional fit which should describe the overall behaviour of the data set. The values of the parameters obtained from the Landau fit are used as initial values in a second fit which uses a Crystal Ball function [49]. In the absence of signal the Crystal Ball function is expected to describe the sample behavior in an ideal way. The obtained curve is shown with a dashed black line in figure 6(a). If the signal hypothesis is true, some mismatch between the fitted function and the histograms is expected. Therefore a new fit is performed to investigate the signal hypothesis. The fit function is the sum of two components: a Crystal Ball term to represent the background and a Breit-Wigner or a Gauss term term to represent the signal 


\begin{tabular}{|r||l|c|c||c|c|c||c|c|c|}
\hline \multicolumn{1}{|c||}{} & \multicolumn{9}{c||}{ LHC $7 \mathrm{TeV}$} \\
\hline selection & limit & $\epsilon_{600}$ & $\epsilon_{\mathrm{BG}}$ & limit & $\epsilon_{900}$ & $\epsilon_{\mathrm{BG}}$ & limit & $\epsilon_{2400}$ & $\epsilon_{\mathrm{BG}}$ \\
\hline min \# of $e / \mu$ & 2 & 92 & 78 & 2 & 95 & 78 & 2 & 98 & 54 \\
\hline$\left|M_{\ell \ell}^{\text {rec }}-M_{Z}\right|<$ & 25 & 95 & 87 & 25 & 95 & 87 & 25 & 54 & 40 \\
\hline$\left|\Delta \phi_{\ell, \ell}\right|<$ & 0.9 & 78 & 26 & 0.7 & 86 & 18 & 0.3 & 82 & 6 \\
\hline min \# of jets & 2 & 92 & 67 & 2 & 92 & 66 & 2 & 93 & 90 \\
\hline$\left|\Delta \eta_{j_{1}, j_{t}}\right|,\left|\Delta \eta_{Z, j_{t}}\right|>$ & 1.4 & 80 & 40 & 1.2 & 86 & 47 & 1.5 & 87 & 48 \\
\hline$p_{T}^{j_{1}}>$ & 180 & 89 & 37 & 250 & 91 & 16 & 610 & 84 & 13 \\
\hline$E_{j_{t}}>$ & 180 & 83 & 54 & 220 & 83 & 46 & 380 & 86 & 51 \\
\hline$\eta_{j_{t}}>$ & 1.3 & 97 & 96 & 1.8 & 90 & 87 & 0.8 & 100 & 99 \\
\hline overall & & 36 & 0.92 & & 41 & 0.25 & & 26 & 0.034 \\
\hline
\end{tabular}

Table 3. Selection criteria and the efficiencies (in percent) for the signal $\left(\epsilon_{m_{Q}}\right)$ and background $\left(\epsilon_{\mathrm{BG}}\right)$ for the case of the neutral current channel with the $Z$ boson decaying to electrons or muons. Note that in evaluating $\epsilon_{\mathrm{BG}}$ all the relevant backgrounds have been included.
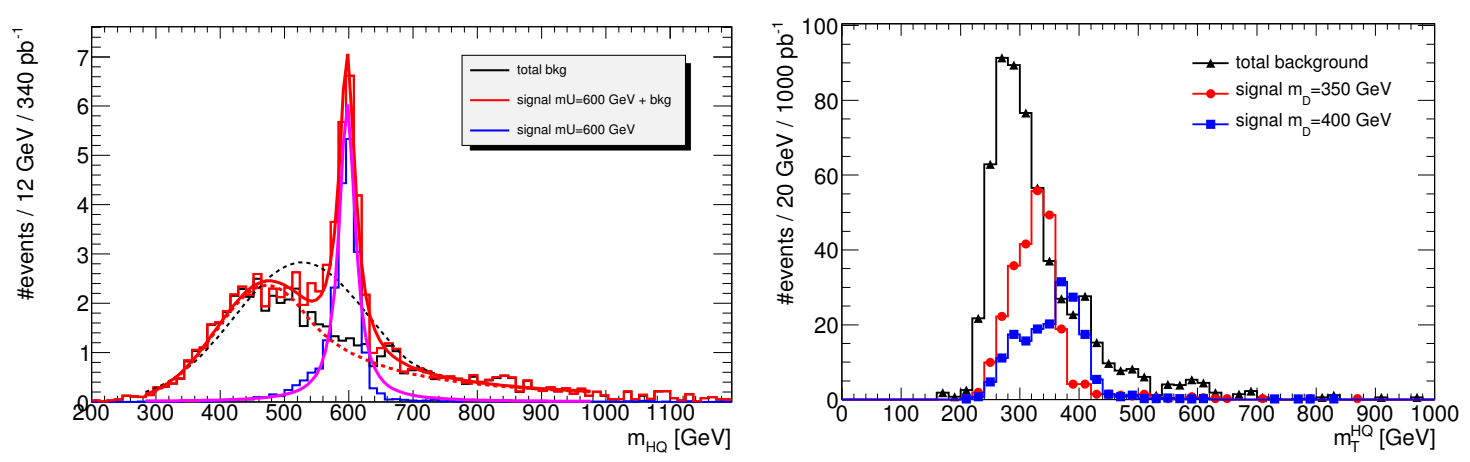

Figure 6. Reconstructed heavy quark distributions at the LHC with $\sqrt{s}=7 \mathrm{TeV}$. (a) Left: invariant mass of the heavy quark of $U$ type and mass $600 \mathrm{GeV}$ decaying leptonically via $\mathrm{NC}$ interactions. See text for a description of all the curves; (b) right: transverse mass of the heavy quark candidates of $D$ type with mass 350 and $400 \mathrm{GeV}$ decaying invisibly into neutrinos via NC interactions (after all the selection criteria have been applied).

resonance. The selection between Breit-Wigner and Gauss terms is made based on the mass of the sought heavy quark: up to $1 \mathrm{TeV}$, the signal is narrow, thus best represented by the former and after $1 \mathrm{TeV}$, the signal distribution becomes fatter thus compatible with the latter. The obtained curve is shown with a red solid line in the invariant mass distributions of figure 6(a). The background and signal components of this function are also shown on the same plot as dashed red and solid magenta lines, respectively. Therefore a good match between the signal (solid blue histogram) and its estimation (solid magenta line) shows the power of the method described above.

Finally, we mention some details about the signal simulation sample. We have simulated signal samples for a $D$ type quark for the $7 \mathrm{TeV}$ analysis and a $U$ type quark for the $14 \mathrm{TeV}$ analysis with $\tilde{\kappa}=1$ and $\operatorname{Br}(Q \rightarrow q Z)=100 \%$. While the efficiencies we list in table 3 are from one quark sample for each c.m. energy, we have checked that there is no 


\begin{tabular}{|r||l|c|c|c|c|}
\hline \multicolumn{1}{|c||}{} & \multicolumn{5}{c|}{ LHC $7 \mathrm{TeV}$} \\
\hline selection & limit & $\epsilon_{350}$ & $\epsilon_{400}$ & $\epsilon_{Z_{\nu \nu}+\text { jets }}$ & $\epsilon_{W_{\ell \nu}+\text { jets }}$ \\
\hline \# of $e / \mu<$ & 1 & 99.5 & 99.4 & 99.5 & 10 \\
\hline tr $_{T}>$ & 100 & 73 & 81 & 13 & 11 \\
\hline min \# of jets & 2 & 92 & 92 & 62 & 65 \\
\hline$p_{T}^{j_{1}}>$ & 100 & 91 & 94 & 63 & 59 \\
\hline$p_{T}^{j_{2}}>$ & 40 & 77 & 80 & 58 & 52 \\
\hline$\left|\eta_{j_{2}}\right|>$ & 1.0 & 74 & 73 & 51 & 57 \\
\hline$\left|\Delta \eta_{j_{1}, j_{2}}\right|>$ & 3.0 & 45 & 46 & 15 & 19 \\
\hline$E_{j_{2}}>$ & 100 & 99 & 99 & 97 & 99 \\
\hline$\left|\Delta \phi_{j_{1}, j_{2}}\right|<$ & 2.7 & 86 & 84 & 82 & 87 \\
\hline$\left|\Delta \phi_{p_{T}, j_{k=1,2,3}}\right|>$ & 0.5 & 79 & 78 & 73 & 59 \\
\hline overall & & 10.5 & 12.1 & 0.13 & 0.01 \\
\hline
\end{tabular}

Table 4. Selection criteria and the efficiencies (in percent) for the signal and background for the case of the neutral current channel with the $Z$ boson decaying to neutrinos. Note that $j_{2}$ here is the same as $j_{t}$.

significant change in the efficiencies while using the other quark sample at the same energy. Note that the choice of the quark sample and the specific model parameters used does not affect the model-independent quantity $S^{Z}$ inferred from an excess of signal events over the background at a given luminosity. The choice of the quark sample does however affect the luminosity required for evidence or discovery of such a quark and the constraints on the various model parameters inferred from it.

\subsection{Neutral current channel with $Z$-decays to neutrinos}

In this section we study the case where the heavy quark decays into a jet and a $Z$ boson, with the $Z$ boson decaying invisibly into neutrinos. An accurate reconstruction of the neutral channel in the $Z_{\nu \nu}+$ jets final state is beyond the scope of this paper, largely due to difficulties in simulating the measurements of large missing transverse energy. Even with detailed GEANT4 simulations of the detectors, fake $E_{T}^{\text {miss }}$ from event mismeasurements (due to non-Gaussian tails in the detector jet responses) is unlikely to be determined with confidence before studies are done with control samples in actual LHC data. However, this final state can still offer some supplementary evidence in the heavy quark searches as we show in this subsection. As an example, we consider the discovery potential for two relatively-low quark masses (350 and $400 \mathrm{GeV}$ ) with $1 \mathrm{fb}^{-1}$ of data at $7 \mathrm{TeV}$ c.m. energy and describe the analysis in detail below.

After all the selection criteria (as listed in table 4), the dominant background is found to be the irreducible background from the Standard Model $Z_{\nu \nu}+$ jets processes. A subdominant contribution is from the $W_{\ell \nu}+$ jet events, where the charged lepton falls outside of the detector acceptance or is lost in the reconstruction. We also consider semileptonic $t \bar{t}$ and jet-associated single top as other sources of background and find them essentially negligible. 
As can be seen in the table, the requirements on $|\Delta \phi|$ between the two leading jets and between any of the three leading jets and the missing transverse momentum appear to play no significant role in separating the signal from the main sources of background. However, we prefer to apply these cuts, since in ref. [50] they have been shown to suppress backgrounds from QCD processes. All the cuts are tested on Pythia di-jet samples generated at various hard scattering momenta, and while we fail to reproduce the results from this reference due to limitations of the fast simulation, we observe that the cuts on the tag jet also provide an extra handle in reducing the QCD backgrounds to negligible levels. Note that for this channel, the tag jet also turns out to be the jet with the second highest transverse momentum i.e. $j_{t} \equiv j_{2}$ and the tag jet is denoted as $j_{2}$ in this section.

Attributing the total missing momentum in the event to the $Z$ boson, the transverse mass of the heavy quark candidate is reconstructed as shown in figure 6(b). While it is clear that the signal and background distributions have distinct shapes and therefore the signal could be extracted using a fitting technique, we conservatively choose to do a simple counting measurement for this final state. Since most of the background is from the $Z_{\nu \nu}+$ jets and to a lesser extent from the $W_{\ell \nu}+$ jet events, there are natural control samples obtained by reverting the charged lepton veto done at the beginning of the event selection. For example $Z_{\ell \ell}+$ jets events can be used to determine the amount of $Z_{\nu \nu}+$ jets background. Therefore for an estimate of the expected significance, we calculate $s / \sqrt{b^{\prime}}$, where $s$ is the expected number of signal events and $b^{\prime}$ includes the expected uncertainty in the background estimation. For simplicity, if we assume $b^{\prime}=b \times \mathrm{BR}(Z \rightarrow \nu \nu) / \mathrm{BR}(Z \rightarrow \ell \ell)$, where $\ell=e, \mu$ and $b$ is the expected total number of background events, we find that the significance for the 350 (400) GeV quark signal is about $6.0(4.2) \sigma$ with $1 \mathrm{fb}^{-1}$ of data.

Next we give some details about the signal simulation sample. We have simulated signal samples for $D$ type quarks with $\tilde{\kappa}=1$ and $\operatorname{Br}(D \rightarrow d Z)=100 \%$. From the studies of the neutral current channel decaying to charged leptons and the charged current channel decaying leptonically, we have found that there is no significant change in the efficiencies while using a $U$ quark sample. Hence, using the same efficiencies as listed in table 4 for a $U$ type quark, which has a larger production cross section compared to the $D$ type quark, would have resulted in a smaller luminosity required for evidence or discovery. For simplicity, we only give the results from the $D$ type quark as an illustration that this channel is a viable mode for evidence or discovery, without loss of generality, as discussed in the previous section.

\subsection{Charged current channel}

In this section we study the case where the heavy quark decays into a jet and a $W$ boson, with the $W$ boson decaying leptonically $W \rightarrow \ell \nu(\ell=e, \mu)$, and investigate the prospects of the LHC in this channel. Once again, we adopt the mass parameters as in eq. (5.4), and describe the analysis in detail below.

The simplest way of reconstructing the $\mathrm{W}$ boson candidate in the charged channel is via its leptonic decays and hence the reconstruction algorithm starts with the selection of events containing at least one electron or muon $(\ell)$ with the selection criteria described in eq. (5.3). In the case where both flavours of leptons are available, the lepton with 
the higher transverse momentum is selected as the $\mathrm{W}$ decay product candidate. After selecting events with a minimum missing transverse energy $\left(\mathbb{F}_{T}\right)$, the four momentum of the $W$ boson is obtained using the information from $\mathscr{E}_{T}$ and $p_{T}^{\ell}$ and including the $\mathrm{W}$ rest mass approximation. The two-fold ambiguity in the solution of the $\mathrm{W}$ boson longitudinal momentum is handled by choosing the most central (minimum $\left|\eta_{W}\right|$ ) solution. We find that, while this method is only slightly better than a random choice at very high quark masses, it reconstructs the true momentum about $65 \%$ of the time for low heavy quark masses, and improves the resolution of the reconstructed heavy quark mass. The azimuthal angle between the charged and neutral lepton is required to be smaller than a threshold (again optimized for each mass point for the best signal significance) to select the boosted $W$ bosons.

The event is required to contain at least two jets isolated from leptons and other jets, the criteria for which are listed in eq. (5.3). The jet with the highest transverse momentum is assumed to be the decay product of the heavy quark. We identify the tag jet as the jet with the highest energy deposition in the event that is at least a minimum $|\Delta \eta|$ apart from both objects that we use to reconstruct the heavy quark candidate i.e. the tag jet has to be well separated from both the reconstructed $W$ boson and the highest $p_{T}$ jet.

We introduce a new variable called "sum $p_{T}$ " which is defined as the absolute value of the vector sum of the tagged jet and the reconstructed heavy quark transverse momenta to enhance the signal over backgrounds,

$$
p_{T}^{\text {sum }} \equiv\left|\vec{p}_{T}^{j_{t}}+\vec{p}_{T}^{Q}\right|
$$

At the leading order, this variable is zero for the signal but detector effects and initial state radiation smear this distribution. However the distribution peaks at very small values for the signal while it is typically large for busy background events, in particular top pair production. We require the $p_{T}^{\text {sum }}$ to be smaller than a threshold to enhance the signal over the backgrounds. This cut reduces the top pair background significantly for the $14 \mathrm{TeV}$ scenario while also having a reasonable impact for the $7 \mathrm{TeV}$ analysis. The efficiencies of these selection criteria in the order they are applied for various signal and background samples are shown in table 5 for the $7 \mathrm{TeV}$ and $14 \mathrm{TeV}$ c.m. energy scenarios and different mass values of the heavy quark. The superscript in the efficiencies of the backgrounds (for e.g. $i$ in $\epsilon_{\mathrm{BG}}^{i}$ ) correspond to $W+$ jets, diboson, top pair and single top for $i=0,1,2,3$ respectively.

The leading jet and the $W$ boson are combined to obtain the reconstructed invariant mass of the heavy quark candidate. The procedure to extract the signal significance is the same as described earlier in section 5.1. The invariant mass distribution for a heavy quark of $D$ type and mass $900 \mathrm{GeV}$ decaying leptonically via $\mathrm{CC}$ channel is shown in figure 7 . In figure 7 (a) we show the reconstructed heavy quark where the charge of the lepton is not identified and in figure 7(b) when the charge of the negatively charged lepton coming from the decay of the heavy quark is identified. When we do not identify the charge of the lepton, we have to include signals from both quark and anti-quark and the background would include the contribution from both $W^{ \pm}+$jets. This method adds a small component to the signal cross section as the anti-quark cross sections are much smaller than quark 


\begin{tabular}{|c|c|c|c|c|c|c|c|c|c|c|c|c|c|c|c|c|c|c|}
\hline & \multicolumn{12}{|c|}{ LHC $7 \mathrm{TeV}$} & \multicolumn{6}{|c|}{ LHC $14 \mathrm{TeV}$} \\
\hline selection & limit & $\epsilon_{600}$ & $\epsilon_{\mathrm{BG}}^{0}$ & $\epsilon_{\mathrm{BG}}^{1}$ & $\epsilon_{\mathrm{BG}}^{2}$ & $\epsilon_{\mathrm{BG}}^{3}$ & |limit & $\epsilon_{900}$ & $\epsilon_{\mathrm{BG}}^{0}$ & $\epsilon_{\mathrm{BG}}^{1}$ & $\epsilon_{\mathrm{BG}}^{2}$ & $\epsilon_{\mathrm{BG}}^{3}$ & limit & $\epsilon_{3000}$ & $\epsilon_{\mathrm{BG}}^{0}$ & $\epsilon_{\mathrm{BG}}^{1}$ & $\epsilon_{\mathrm{BG}}^{2}$ & $\epsilon_{\mathrm{BG}}^{3}$ \\
\hline $\begin{array}{c}\min \# \\
\text { of } e / \mu\end{array}$ & 1 & 96 & 91 & 91 & 94 & 92 & 1 & 97 & 91 & 91 & 94 & 92 & 1 & 99 & 98 & 90 & 96 & 96 \\
\hline$E_{T}>$ & 30 & 94 & 76 & 70 & 80 & 80 & 50 & 92 & 57 & 35 & 54 & 52 & 110 & 96 & 62 & 8 & 39 & 65 \\
\hline$\nu$ solution & & 82 & 80 & 74 & 73 & 74 & & 81 & 80 & 70 & 71 & 71 & & 67 & 78 & 76 & 77 & 77 \\
\hline$\left|\Delta \phi_{\ell, \nu}\right|<\mid$ & 0.8 & 86 & 41 & 13 & 31 & 19 & 0.6 & 91 & 29 & 13 & 25 & 14 & 0.5 & 99 & 84 & 50 & 63 & 71 \\
\hline $\begin{array}{l}\min \# \\
\text { of jets }\end{array}$ & 2 & 91 & 63 & 89 & 100 & 95 & 2 & 90 & 63 & 90 & 100 & 95 & 2 & 90 & 96 & 95 & 99 & 95 \\
\hline $\begin{array}{l}\left|\Delta \eta_{j_{t}, W}\right| \\
\left|\Delta \eta_{j_{t}, j_{1}}\right|>\end{array}$ & 1.8 & 68 & 28 & 20 & 30 & 28 & 2 & 65 & 24 & 17 & 25 & 23 & 2.3 & 72 & 31 & 25 & 32 & 30 \\
\hline $\overrightarrow{p_{T}^{j_{1}}>}$ & 200 & 82 & 25 & 26 & 18 & 15 & 250 & 90 & 17 & 24 & 14 & 11 & 450 & 98 & 67 & 53 & 24 & 70 \\
\hline$E_{j_{t}}>$ & 200 & 78 & 61 & 60 & 71 & 62 & 300 & 74 & 52 & 47 & 60 & 52 & 270 & 95 & 72 & 73 & 79 & 70 \\
\hline$\left|\eta_{j_{t}}\right|>\mid$ & 0.6 & 100 & 100 & 100 & 100 & 100 & 0.6 & 100 & 100 & 100 & 100 & 100 & 0.6 & 100 & 100 & 100 & 100 & 100 \\
\hline$p_{T}^{\text {sum }}$ & 100 & 94 & 76 & 56 & 47 & 47 & 100 & 93 & 74 & 56 & 41 & 40 & 100 & 74 & 48 & 38 & 18 & 18 \\
\hline$Q_{\ell}=-1$ & & 85 & 31 & 39 & 49 & 28 & & 88 & 31 & 50 & 47 & 36 & & 93 & 29 & 45 & 45 & 29 \\
\hline overall & & 20 & 0.15 & 0.037 & 0.15 & 0.031 & & 21 & 0.036 & 0.014 & 0.035 & 0.0089 & & 26 & 0.82 & 0.041 & 0.088 & 0.25 \\
\hline$Q_{\ell}=+1$ & & 90 & 69 & 61 & 51 & 72 & & 94 & 69 & 50 & 53 & 64 & & 97 & 71 & 55 & 55 & 71 \\
\hline overall & & 24 & 0.32 & 0.059 & 0.16 & 0.079 & & 25 & 0.083 & 0.014 & 0.039 & 0.016 & & 29 & 1.99 & 0.050 & 0.11 & 0.60 \\
\hline
\end{tabular}

Table 5. Selection efficiencies (in percent) for signal $\left(\epsilon_{m_{Q}}\right)$ and background $\left(\epsilon_{\mathrm{BG}}^{i}\right)$ samples for the case of the charged current channel with leptonic decays into electrons and muons. The superscript in the efficiencies of the backgrounds (for e.g. $i$ in $\epsilon_{\mathrm{BG}}^{i}$ ) correspond to $W+$ jets, diboson, top pair and single top for $i=0,1,2,3$ respectively.
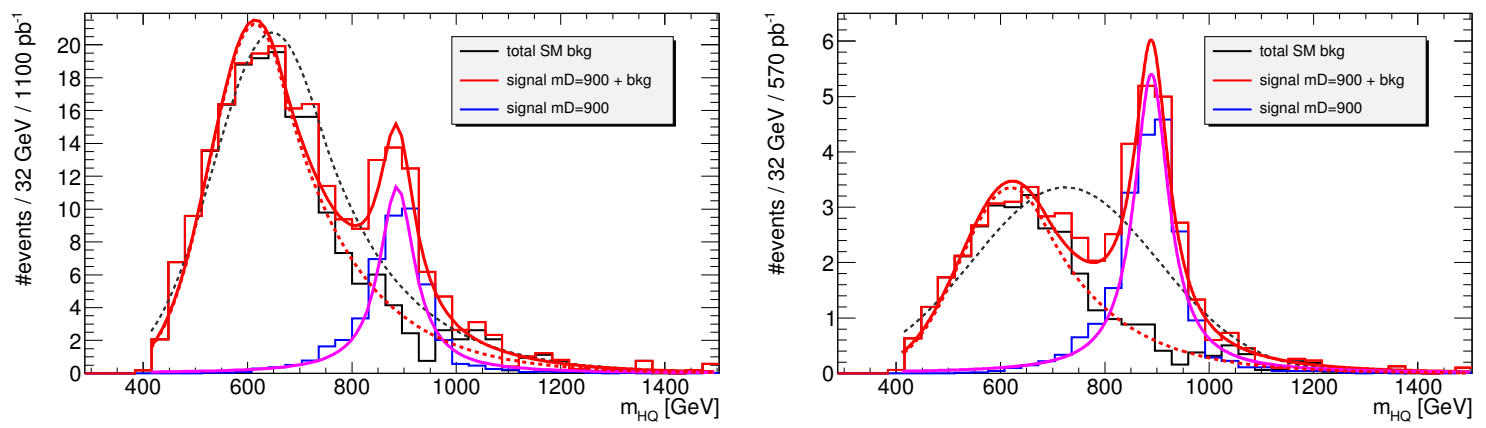

Figure 7. Reconstructed heavy quark distributions for a quark of type $D$ and mass $900 \mathrm{GeV}$ decaying via $\mathrm{CC}$ interactions at the LHC with $\sqrt{s}=7 \mathrm{TeV}$. (a) Left: no charge identification of the charged lepton has been made; (b) right: with charge identification of the negatively charged lepton. See section 5.1 for a description of all the curves.

cross sections while adding significantly to the backgrounds as positively charged $W$ bosons are produced preferentially at a $p p$ collider such as the LHC. This reduces the signal significance. However identifying the charge of the negative lepton enhances the signal over the backgrounds and reduces the luminosity required for discovery by almost half. For the heavy quarks decaying into positively charged leptons (say $U$ and $X$ ), again the anti-quark contributions add very little to the signal but the reduction in the background is 

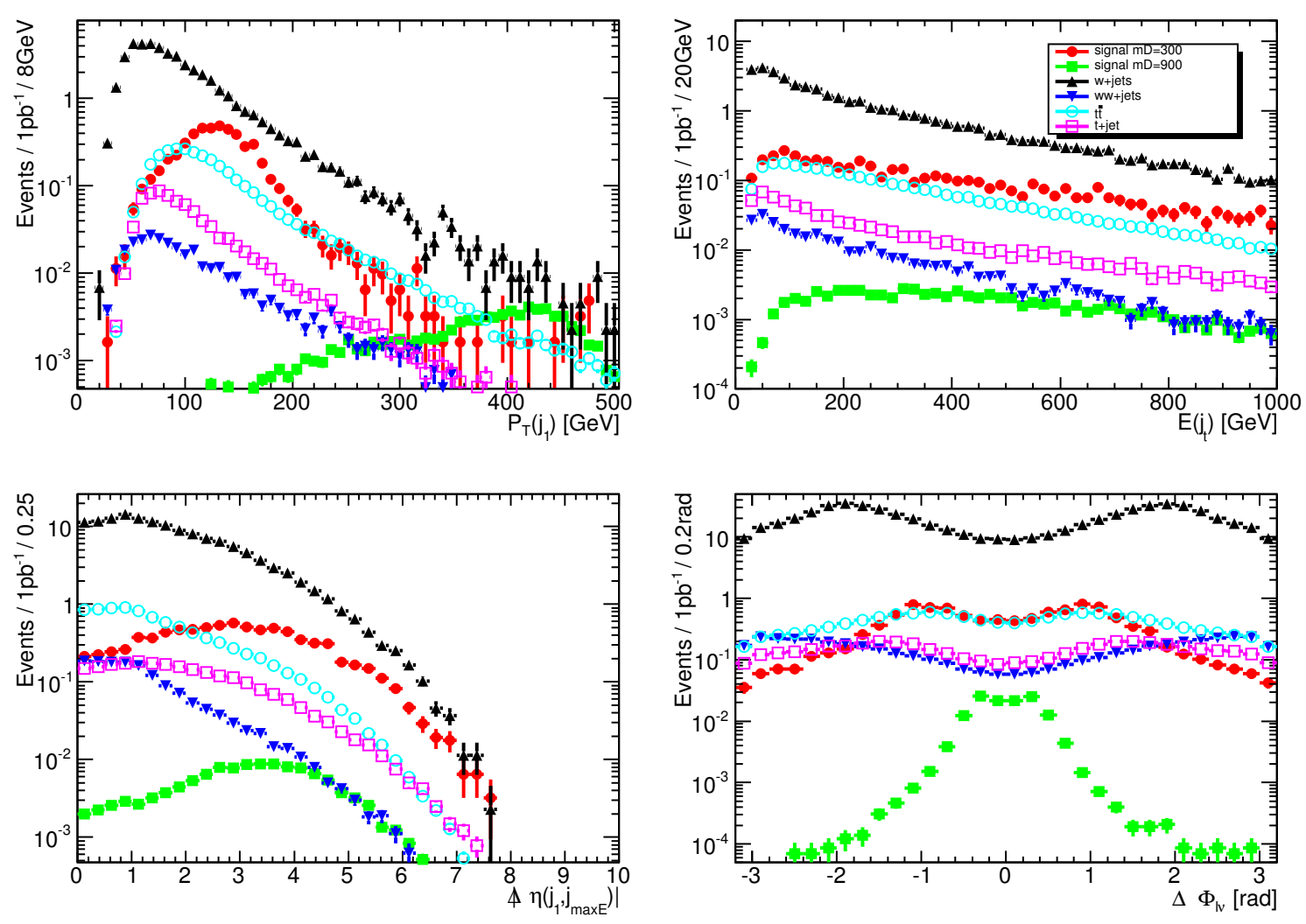

Figure 8. (a) Top left: $p_{T}$ of the hardest jet. This jet is used in reconstructing the heavy quark mass; (b) top right: total energy of the forward, tagged jet (c) bottom left: difference in pseudorapidity between the hardest jet and the tagged jet $(\mathrm{d})$ bottom right: azimuthal angle between $p_{T}(\ell)$ and $p_{T}$.

much smaller as $W^{+}$are produced preferentially at the LHC. However, there is still some improvement in the signal significance by identifying the charge of the positive lepton though not as much as in the case of the negative leptons. The effect of identifying the charge of the lepton (for both positive and negatively charged leptons) is shown in table 5 .

We show some sample distributions for a few of the relevant kinematic quantities which are unique to single production of heavy quarks and enable us to separate the signal from the background in figure 8 . The $p_{T}$ distribution of the hard jet and the energy distribution of the forward tagged jet are shown in figure 8(a) and figure 8(b) respectively. The difference in pseudo-rapidity between the hard jet and the forward tagged jet is shown in figure 8(c) and the azimuthal angle separation between the leptons $\left(p_{T}^{\ell}\right.$ and $\left.p_{T}\right)$ is shown in figure $8(\mathrm{~d})$. All distributions in figure 8 are normalized to $1 \mathrm{pb}^{-1}$ integrated luminosity.

We mention some details about the signal simulation sample. We simulate signal samples for both $D$ and $U$ type quarks with $\tilde{\kappa}=1$ and $\operatorname{Br}(Q \rightarrow q W)=100 \%$. The efficiencies we list for the various cuts (except the charge identification cut) in table 5 are from the $D$ quark sample. However, we have checked that there is no significant change in the efficiencies while using a $U$ quark sample, except for the cut on $E_{j_{t}}$ where a $U$ type 
quark has a higher signal efficiency compared to the $D$ type quark. The reason is as follows: from eq. (3.1) we see that the dominant contribution comes from the case where the tag jet is a SM $d$ quark for $D$ type quark and a SM $u$ quark for the $U$ type quark. Due to the PDFs, the $u$ quark has a slightly harder energy distribution compared to the $d$ quark, which results in a higher efficiency for the tag jet in the case of the signal with $U$ type quark compared to the $D$ type quark. While the numbers we list in table 5 are for the $D$ type quark we use the actual values for the $U$ type quark when extracting the signal and background events for evidence or discovery reach.

In the last few rows we show the efficiency when the charge of the lepton coming from the decay of the heavy quark is identified. For $Q_{\ell}=-1$ we use the $D$ type quark sample and for $Q_{\ell}=+1$ we use the $U$ type quark sample. Note that the efficiency for identifying a lepton correctly as positively or negatively charged is the same irrespective of origin of the lepton. In fact in our analysis the efficiency of identifying the charge of a lepton itself is essentially $100 \%$ (see section 6 for details). However from table 5 it is evident that a $U$ type quark has a slightly higher efficiency in identifying the charge of the lepton. The reason is that we start with a $Q+\bar{Q}$ sample and then identify the charge of the positive or negatively charged leptons in this sample. Given that $U$ type quark has a smaller antiquark component (which gives the wrong sign leptons) of the total cross section compared to the $D$ type quark, we get a slightly higher efficiency for charge identification for the $U$ type quark.

Finally, note that the choice of the quark sample and the specific model parameters used does not affect the model-independent quantity $S^{W^{ \pm}}$inferred from an excess of signal events over the background at a given luminosity. The model-independent parameterization discussed in section 4 was designed such that the effects of $\tilde{\kappa}$ and the branching ratio of the heavy quark decay mode could be factored out. The choice of the quark sample does however affect the luminosity required for evidence or discovery of such a quark and the constraints on the various model parameters inferred from it.

\subsection{Identification of the heavy quark}

In this section we discuss the interesting possibility of determining the identity of the heavy quark produced. In $p \bar{p}$ collisions such as at the Tevatron, this is rather straight forward as demonstrated in ref. [4]: defining the proton beam as the $+\hat{z}$ direction, the forward (backward) tagged jet should be correlated with the heavy anti-quark (quark) production. Moreover, this can be used as an indication for down-type or up-type heavy quark production by identifying the electromagnetic charge of the lepton from the charged current decay. For e.g., an event with a backward tagged jet and a positive (negative) lepton would indicate production of $U(D)$ heavy quark. Similarly, an event with a forward tagged jet and a positive (negative) lepton would indicate production of $\bar{D}(\bar{U})$ heavy quark. However, note that one cannot distinguish between a charge $2 / 3$ and charge $5 / 3$ quark (or a charge $-1 / 3$ and charge $-4 / 3$ quark) by this procedure.

At a $p p$ machine such as the LHC, the situation is somewhat different. First of all, as seen from the total cross section curves in figures $2-4$, a heavy quark production is substantially larger than that for an anti-quark, due to the $u, d$ valence quark dominance. 
There is a further subtle difference between the production of a heavy quark and an antiquark. A valence quark parton has a harder distribution in the energy fraction $x$, while an anti-quark parton distribution is a lot softer. Consequently, a heavy quark produced will be more strongly boosted. Finally, the leptonic decay of a heavy quark via charged-current may still be utilized to specify the electric charge of $U$ or $D$.

Another way to exploit the difference in the cross section of a heavy quark and antiquark to gain sensitivity to the charge of the heavy quark produced is described below. We define a charge asymmetry which will be independent of the couplings $\tilde{\kappa}$ or of the branching ratios:

$$
A=\frac{N_{+}-N_{-}}{N_{+}+N_{-}},
$$

where $N_{ \pm}$is the cross section measured for the $W^{ \pm} j$ channel. For example, at $\sqrt{s}=7 \mathrm{TeV}$, based on the values given in table 1 , one finds $A=(-0.77,0.84,0.95,-0.41)$ for quarks of mass $900 \mathrm{GeV}$ and of type (D,U,X,Y).

Note however, that both of the methods described above work best when the quarks are non-degenerate and the resonances can be distinguished experimentally. In that case, the charge asymmetry and/or the rapidity of the reconstructed quark along with the charge of the observed lepton could serve to distinguish between the quarks with different electromagnetic charges. When the states are exactly degenerate (equal mass and equal couplings) these methods have a contamination from the anti-quarks from a few percent up to few tens of percent depending on the model and the heavy quark under consideration. For example, in the case of doublets with hypercharges $1 / 6$ and $7 / 6$, upon observing a $5 \sigma$ excess in the $S^{W-}$ channel for a mass of $900 \mathrm{GeV}$, one would have a contamination from the $\bar{X}$ quark $\sim 5 \%$ in the case of degenerate bidoublets. If the quarks were non-degenerate with couplings also varying by $10 \%$, then the contamination is $\mathcal{O}(10 \%)$. If the observation were to be in the $S^{W+}$ channel for the hypercharge $1 / 6$ and $-5 / 6$ doublets, the contribution from the anti-quarks increases to $\sim 20 \%$ for the degenerate case and further goes up to $\mathcal{O}(30 \%)$ for close by resonances with slightly unequal couplings. In summary, the methods described for charge identification are best applied for the case of non-degenerate quarks and caution must be exercised when using the same methods for degenerate (and very close in mass and coupling) quarks.

\section{Results}

In this section we will consider the current constraints from direct searches, the sensitivity of the search for new heavy vector-like quarks at the LHC, the constraints on some example models at the LHC and finally discuss some systematic effects.

\subsection{Current constraints from direct searches}

The current bound from direct searches at the Tevatron experiments is

$$
m_{Q}>335(268) \mathrm{GeV} \text { at } 95 \% \text { C.L. }
$$

for heavy up (down) type quarks produced in pairs and decaying via $\mathrm{CC}(\mathrm{NC})$ interactions [51, 52]. Recently both CDF and D0 experiments at the Tevatron did a search for 
heavy quarks with sizable couplings to first generation SM quarks in the single production channel $[53,54]$. They presented their results in a model-independent fashion in the plane of mass of the heavy quark vs cross section times branching ratio (a parameter similar to $S^{V}$ ). From these analyses the heavy quarks have limits as given below.

$$
\begin{array}{rll}
\mathrm{CDF}: & \sigma(p \bar{p} \rightarrow q Q) \times \operatorname{Br}(Q \rightarrow q W) \lesssim 240 \mathrm{fb} & \text { for } m_{Q}=530 \mathrm{GeV} \\
\mathrm{D} 0: & \sigma(p \bar{p} \rightarrow q Q) \times \operatorname{Br}(Q \rightarrow q W) \lesssim 40 \mathrm{fb} & \text { for } m_{Q}=690 \mathrm{GeV} \\
\mathrm{D} 0: & \sigma(p \bar{p} \rightarrow q Q) \times \operatorname{Br}(Q \rightarrow q Z) \lesssim 120 \mathrm{fb} & \text { for } m_{Q}=550 \mathrm{GeV}
\end{array}
$$

where $Q$ is a generic heavy quark. The charge of the lepton was not identified in these analyses and hence the correlation with our $S^{V}$ parameters is slightly different and is given by

$$
\begin{aligned}
\sigma(p \bar{p} \rightarrow q Q) \times \operatorname{Br}(Q \rightarrow q W) & =S^{W^{-}}+S^{W^{+}} & & \text {for CC } \\
\sigma(p \bar{p} \rightarrow q Q) \times \operatorname{Br}(Q \rightarrow q Z) & =S^{Z} & & \text { for } \mathrm{NC} .
\end{aligned}
$$

The Tevatron being a $p \bar{p}$ machine, heavy quarks and anti-quarks are produced in equal numbers with the result that $S^{W^{-}}=S^{W^{+}}$. The constraints from the Tevatron (on $S^{W^{+}}+S^{W^{-}}$) can hence be translated into constraints on $S^{W^{+}}$or $S^{W^{-}}$.

While it is very useful to have model-independent limits due to the wide applicability of results, it is also illustrative to interpret the results in the case of a particular model. For example, interpreting these results in the context of degenerate doublets (with hypercharges $1 / 6$ and $7 / 6$ ) with $\tilde{\kappa}_{u D}=1$ and decaying $100 \%$ via CC currents, the results from CDF exclude heavy $D$ type quarks up to mass $530 \mathrm{GeV}$ while the D0 constraints are more stringent and exclude heavy $D$ type quarks up to $690 \mathrm{GeV}$. In the same model for a $U$ type quark with $\tilde{\kappa}_{u U}=\sqrt{2}$ and decaying $100 \%$ via NC currents, the sensitivity is to a $550 \mathrm{GeV}$ quark. Note that in the context of this model, since all the quarks are degenerate, a $U$ type quark of mass $690 \mathrm{GeV}$ decaying via $\mathrm{NC}$ is also excluded. However in other models the interpretation can be quite different.

\subsection{Sensitivity at the LHC}

We have simulated signal and background events for two different scenarios: an early run with $\sqrt{s}=7 \mathrm{TeV}$ and an integrated luminosity of $1 \mathrm{fb}^{-1}$, and a longer run with $\sqrt{s}=14 \mathrm{TeV}$ and $100 \mathrm{fb}^{-1}$ integrated luminosity. The estimated number of signal and background events are extracted using the fitted function to the total number of events as described in section 5.1. The statistical significance is calculated as [55]

$$
\mathcal{S}=\sqrt{2 \times\left[(s+b) \ln \left(1+\frac{s}{b}\right)-s\right]},
$$

where $s(b)$ is the number of signal (background) events determined from the Breit-Wigner (Crystal Ball) term of the fitted function by integrating over $2 \sigma$ of the most probable value of the Breit-Wigner component. The estimated number of signal and background events extracted from the fits to the total number of events for the LHC with $\sqrt{s}=7 \mathrm{TeV}$ and 


\begin{tabular}{|c|c|c|c|}
\hline \multicolumn{5}{|c|}{ Channel: $\ell^{+} \ell^{-} 2 j$} \\
\hline$m_{Q}(\mathrm{GeV})$ & 300 & 600 & 900 \\
\hline Signal events & $10.7(29.2)$ & $7.10(20.3)$ & $6.80(18.9)$ \\
\hline Background events & $9.30(25.4)$ & $3.70(11.0)$ & $3.30(9.20)$ \\
\hline $\int$ Luminosity $\left(\mathrm{pb}^{-1}\right)$ & $22.0(60.0)$ & $116(340)$ & $540(1500)$ \\
\hline \multicolumn{4}{|c|}{ Channel: $\ell^{-} \mathbb{t}_{T} 2 j$} \\
\hline$m_{Q}(\mathrm{GeV})$ & 300 & 600 & 900 \\
\hline Signal events & $12.7(35.3)$ & $7.60(21.1)$ & $6.48(18.0)$ \\
\hline Background events & $14.1(39.3)$ & $4.28(11.9)$ & $2.77(7.70)$ \\
\hline $\int$ Luminosity $\left(\mathrm{pb}^{-1}\right)$ & $6.80(18.9)$ & $37.1(103)$ & $205(570)$ \\
\hline \multicolumn{5}{|c|}{ Channel: $\ell^{+} \not \mathbb{t}_{T}$} & $2 j$ \\
\hline$m_{Q}(\mathrm{GeV})$ & 300 & 600 & 900 \\
\hline Signal events & $17.8(49.4)$ & $12.8(35.5)$ & $8.78(24.4)$ \\
\hline Background events & $29.7(82.5)$ & $14.1(39.1)$ & $6.08(16.9)$ \\
\hline $\int$ Luminosity $\left(\mathrm{pb}^{-1}\right)$ & $11.2(31.1)$ & $75.6(210)$ & $283(785)$ \\
\hline
\end{tabular}

Table 6. The estimated number of signal and background events extracted from the fits to the total number of events for the LHC with $\sqrt{s}=7 \mathrm{TeV}$. For each case, the values for $3 \sigma$ (and $5 \sigma$ in parentheses) statistical significance is also given.

\begin{tabular}{|c|c|c|c|}
\hline \multicolumn{4}{|c|}{ Channel: $\ell^{+} \ell^{-} 2 j$} \\
\hline$m_{Q}(\mathrm{GeV})$ & 900 & 1800 & 2400 \\
\hline Signal events & $10.1(28.3)$ & $8.60(24.1)$ & $13.1(37.0)$ \\
\hline Background events & $8.20(23.0)$ & $5.80(16.3)$ & $15.1(42.8)$ \\
\hline $\int$ Luminosity $\left(\mathrm{fb}^{-1}\right)$ & $0.15(0.42)$ & $2.64(7.40)$ & $35.0(99.0)$ \\
\hline \multicolumn{4}{|c|}{ Channel: $\ell^{-} \mathbb{E}_{T} 2 j$} \\
\hline$m_{Q}(\mathrm{GeV})$ & 900 & 1800 & 3000 \\
\hline Signal events & $9.29(25.8)$ & $4.72(13.1)$ & $4.18(11.6)$ \\
\hline Background events & $6.70(18.6)$ & $1.22(3.40)$ & $0.720(2.00)$ \\
\hline $\int$ Luminosity $\left(\mathrm{fb}^{-1}\right)$ & $0.0450(0.125)$ & $0.360(1.00)$ & $6.12(17.0)$ \\
\hline \multicolumn{4}{|c|}{ Channel: $\ell^{+} \mathbb{E}_{T} 2 j$} \\
\hline$m_{Q}(\mathrm{GeV})$ & 900 & 1800 & 3000 \\
\hline Signal events & $15.7(43.7)$ & $13.1(36.3)$ & $6.48(18.0)$ \\
\hline Background events & $23.6(65.6)$ & $14.4(40.0)$ & $2.41(6.70)$ \\
\hline $\int$ Luminosity $\left(\mathrm{fb}^{-1}\right)$ & $0.0864(0.240)$ & $1.15(3.20)$ & $11.5(32.0)$ \\
\hline
\end{tabular}

Table 7. Same as table 6 but for $14 \mathrm{TeV}$.

$14 \mathrm{TeV}$ are presented in table 6 and table 7 respectively. For each case, the number of signal and background events giving rise to $3 \sigma$ evidence and $5 \sigma$ discovery (in parentheses) are also given. In the same tables are also given the luminosities required for $3 \sigma$ evidence and $5 \sigma$ discovery of heavy quarks corresponding to three different channels, namely, $\ell^{+} \ell^{-} 2 j, \ell^{-} \oiint_{T} 2 j$ and $\ell^{+} \mathscr{E}_{T} 2 j$. While the number of signal and background events are model-independent quantities, the luminosity required is model-dependent. The luminosities shown are for a 

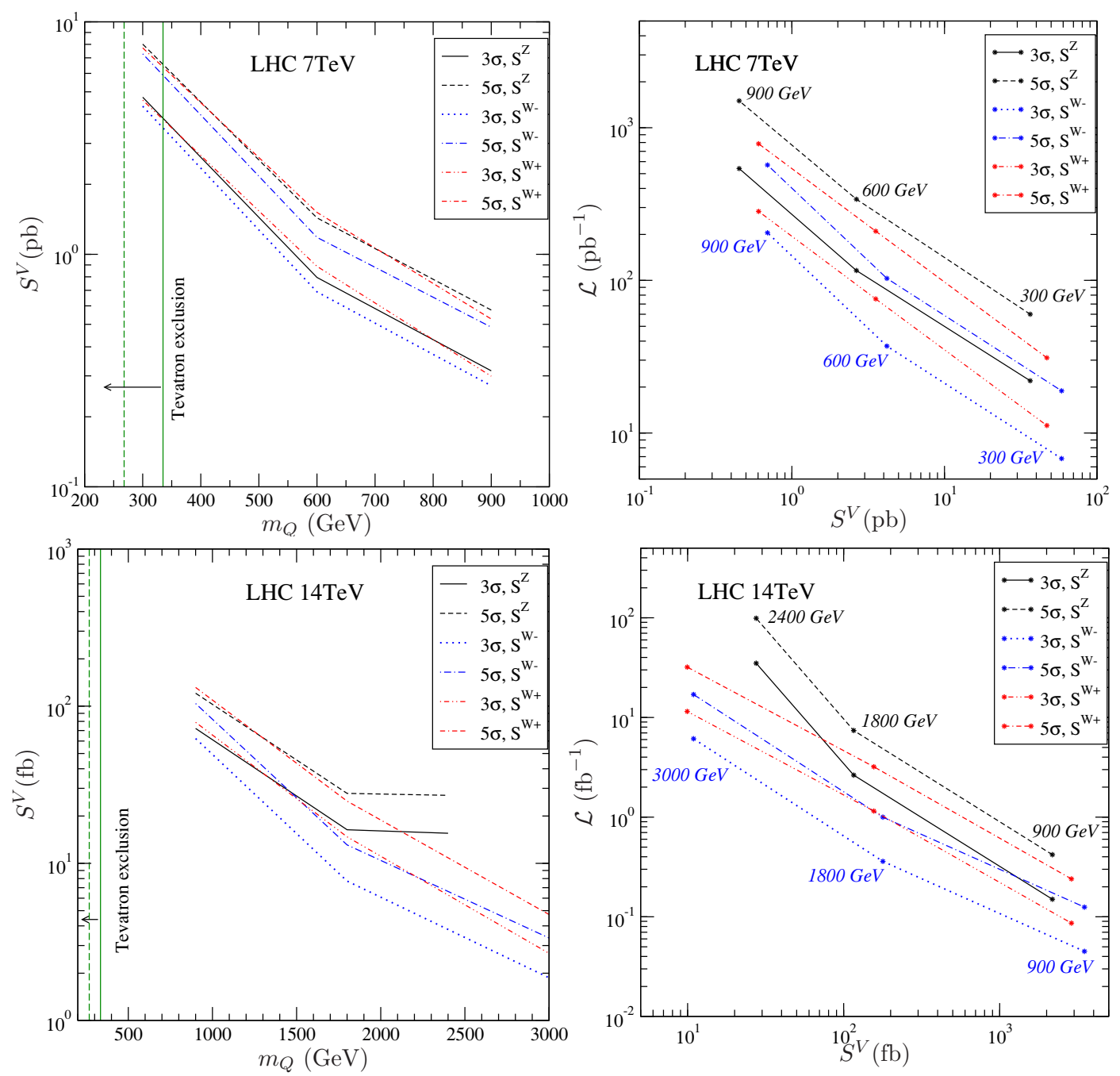

Figure 9. (a) Top left: sensitivity plot for $3 \sigma$ evidence and $5 \sigma$ discovery in the plane of the parameters $S^{W-}, S^{W+}$ and $S^{Z}$ and heavy quark mass $m_{Q}$ with $1 \mathrm{fb}^{-1}$ of data at $\sqrt{s}=7 \mathrm{TeV}$; (b) top right: luminosity needed for $3 \sigma$ evidence and $5 \sigma$ discovery vs the parameters $S^{W-}, S^{W+}$ and $S^{Z}$ at the LHC with $\sqrt{s}=7 \mathrm{TeV}$. The three points on each curve represented by black (blue or red) stars correspond to the mass for each $S^{Z}\left(S^{W^{-}}\right.$or $\left.S^{W^{+}}\right)$; (c) bottom left: same as (a) but for $\sqrt{s}=14 \mathrm{TeV}$ and $100 \mathrm{fb}^{-1}$ of data; (d) bottom right: same as (b) but for $\sqrt{s}=14 \mathrm{TeV}$. The vertical solid green and vertical solid dashed green bounds are the $95 \%$ C.L. exclusion limits from Tevatron for the heavy quarks produced in pairs via QCD interactions and decaying via $\mathrm{CC}$ and $\mathrm{NC}$ decay modes respectively.

$U, D$ and $U$ type quark for the $\ell^{+} \ell^{-} 2 j, \ell^{-} \mathbb{F}_{T} 2 j$ and $\ell^{+} \mathbb{F}_{T} 2 j$ channels respectively. For each of these quarks, the model-dependent parameter, $\tilde{\kappa}=1$ while the decay of the heavy quark in the respective gauge boson mode is $100 \%$. The choice of these parameters is for illustrative purposes only and corresponds to the degenerate bidoublet scenario.

We present the model-independent results of our analysis for the LHC in figure 9 for the different channels. There are three parameters $-S^{V}, m_{Q}$ and the luminosity - in our analysis and we present our results in the planes defined by two of these parameters. In 
figure 9 (a) and figure $9(\mathrm{c})$ we present the sensitivity plot for $3 \sigma$ evidence and $5 \sigma$ discovery in the plane of the parameters $S^{W-}, S^{W+}$ and $S^{Z}$ and heavy quark mass $m_{Q}$ for $\sqrt{s}=7 \mathrm{TeV}$ with $1 \mathrm{fb}^{-1}$ of data and for $\sqrt{s}=14 \mathrm{TeV}$ with $100 \mathrm{fb}^{-1}$ of data respectively. In figure 9 (b) and figure $9(\mathrm{~d})$ we present the luminosity needed for $3 \sigma$ evidence and $5 \sigma$ discovery vs $S^{V}$ at the LHC with $\sqrt{s}=7 \mathrm{TeV}$ and $\sqrt{s}=14 \mathrm{TeV}$ respectively. In figures 9 (b) and (d) the quark masses change along the different curves. For reference we show with stars three different values of quark masses on each curve.

The optimal channel for discovery in an early LHC run is $m_{Q} \approx 1 \mathrm{TeV}$ for $S^{W^{-}} \approx 0.41 \mathrm{pb}$ for $\sqrt{s}=7 \mathrm{TeV}$ and $1 \mathrm{fb}^{-1}$ integrated luminosity. This is comparable to the final Tevatron reach for single production of these quarks. Of course, the reach increases dramatically for a longer LHC run. We have estimated that with $100 \mathrm{fb}^{-1}$ at $14 \mathrm{TeV}$ and $S^{W^{-}}=2.6 \mathrm{fb}$, the LHC can discover a new $Q$ quark with a mass up to $m_{Q} \approx 3.7 \mathrm{TeV}$.

\subsection{Constraints on specific models}

We interpret the model-independent results presented in figure 9 in terms of some specific models next. We present the sensitivity of the model dependent parameter $\tilde{\kappa}_{q Q}$ as a function of the mass of the heavy quark $\left(m_{Q}\right)$ for some popular scenarios. We present the $3 \sigma$ and $5 \sigma$ constraints on $\tilde{\kappa}_{q Q}$ for $\sqrt{s}=7 \mathrm{TeV}$ with $1 \mathrm{fb}^{-1}$ of data and $\sqrt{s}=14 \mathrm{TeV}$ with $100 \mathrm{fb}^{-1}$ of data in figure 10 and figure 11 respectively. We also show the model dependent constraints from electroweak precision observables, which correspond to

$$
\kappa_{q Q} \geq 0.75,0.066,0.041,0.037
$$

for the degenerate bidoublet, doublet, D singlet and U singlet models, respectively. We also show in figure 10 the current constraints from the recent Tevatron searches. Several conclusions can be obtained from our results. First, as expected, only for the degenerate bidoublet model can the Tevatron and the early LHC run probe regions of parameter space which are not excluded by electroweak precision tests. Second, the early LHC run reach is comparable and in general better than the Tevatron reach. In some cases, like the neutral channel in the $U$ singlet model, the Tevatron is unable to constrain the model (the probed cross sections cannot be generated due to the bound from the unitarity of the mixing) whereas they are all probed by the LHC. Finally, the reach of the LHC run at $\sqrt{s}=14 \mathrm{TeV}$ and $100 \mathrm{fb}^{-1}$ integrated luminosity is sensibly better, probing regions of parameter space not excluded by electroweak precision tests for all four models (for large enough masses). In particular, masses in the $3-4 \mathrm{TeV}$ region can be probed in all four models provided we have $\tilde{\kappa}_{q Q} \gtrsim 0.4$. Recall that for these large masses, $\tilde{\kappa}_{q Q}$ can be directly related to a Yukawa coupling in particular models. Next we consider a few example models in detail.

\subsection{1 $\quad D$ type singlet}

The first model contains an extra vector-like electroweak quark singlet with hypercharge $-1 / 3$. We assume the singlet mixes only with the first generation (doublet) in the basis in which all SM flavour occurs in the up quark sector. The model can be fully parameterized 

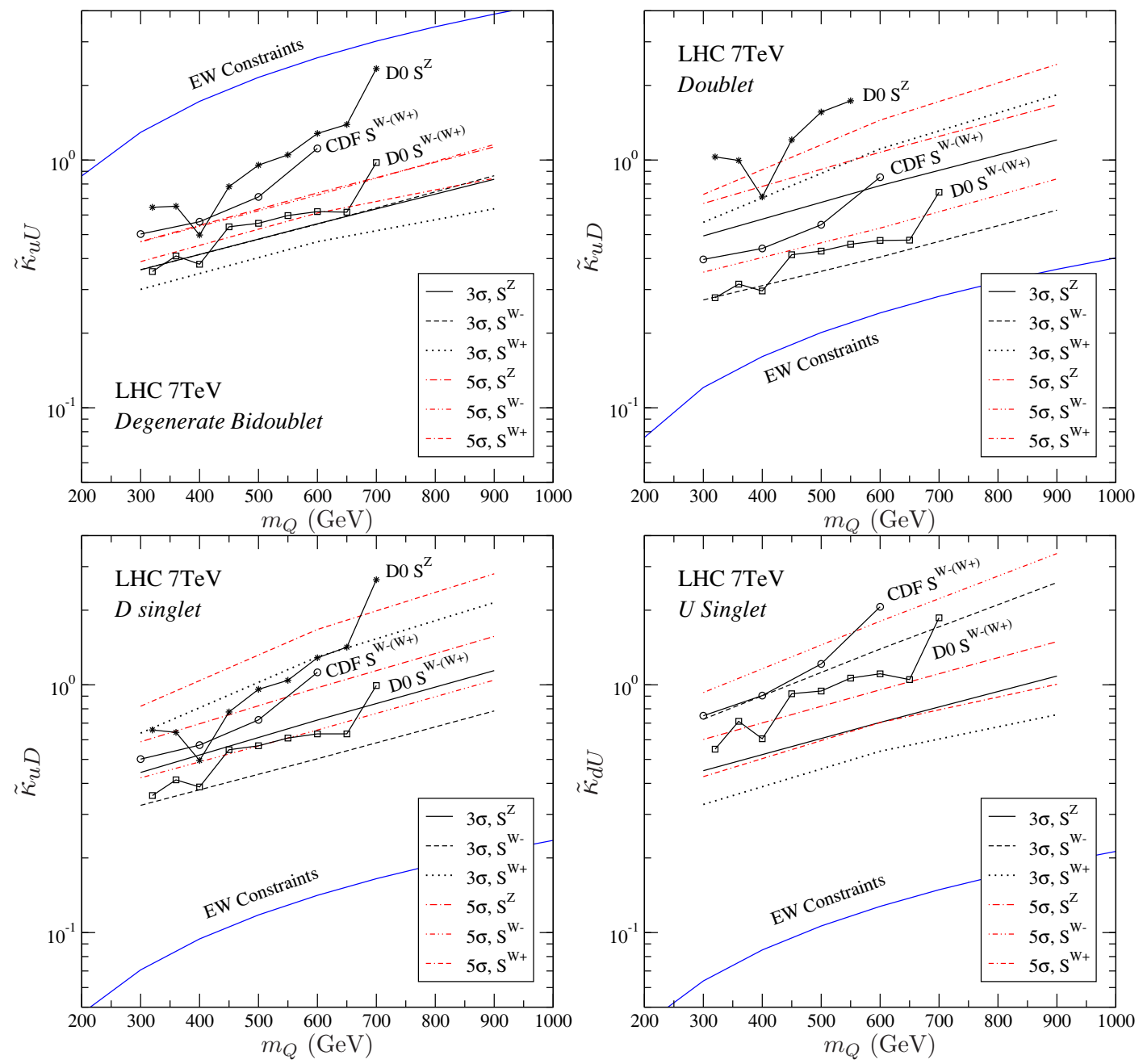

Figure 10. (a) Top left: sensitivity plot for $3 \sigma$ evidence and $5 \sigma$ discovery in the plane of the model parameters $\tilde{\kappa}_{u U}$ and heavy quark mass $m_{Q}$ at the LHC with $1 \mathrm{fb}^{-1}$ of data and $\sqrt{s}=7 \mathrm{TeV}$ for the model with degenerate bidoublets; (b) top right: same as (a) but for $\tilde{\kappa}_{u D}$ for the model with one doublet; (c) bottom left: same as (a) but for $\tilde{\kappa}_{u D}$ for the model with $D$ type singlet (d) bottom right: same as (a) but for $\tilde{\kappa}_{d U}$ for the model with $U$ type singlet. The curves with data points show the $95 \%$ C.L. constraints on $\tilde{\kappa}_{q Q}$ for each model coming from the direct searches by CDF and D0 experiments in the channel where heavy quarks are produced singly via EW interactions. The region above the blue solid line denoted "EW Constraints" is excluded by the EW precision tests described in section 2.2. The bounds on the other couplings in each model can be obtained from the relations between couplings listed in section 6.3 .

in terms of the physical down quark mass, $m_{d}$, the physical heavy down quark mass, $m_{D}$, and the coupling between $D$ and $u, \kappa_{u D}^{L} \equiv V_{u d} s_{L}$, with $V$ the CKM matrix and $s_{L}$ is used to denote the sine of an angle (and is therefore smaller than one). The decoupling limit occurs for $m_{D} \rightarrow \infty, s_{L} \rightarrow 0$ with $s_{L} m_{D}$ constant. In that limit all effects go to zero. The non-zero couplings apart from $\kappa_{u D}^{L}$, are

$$
\kappa_{d D}^{L}=s_{L} c_{L}
$$



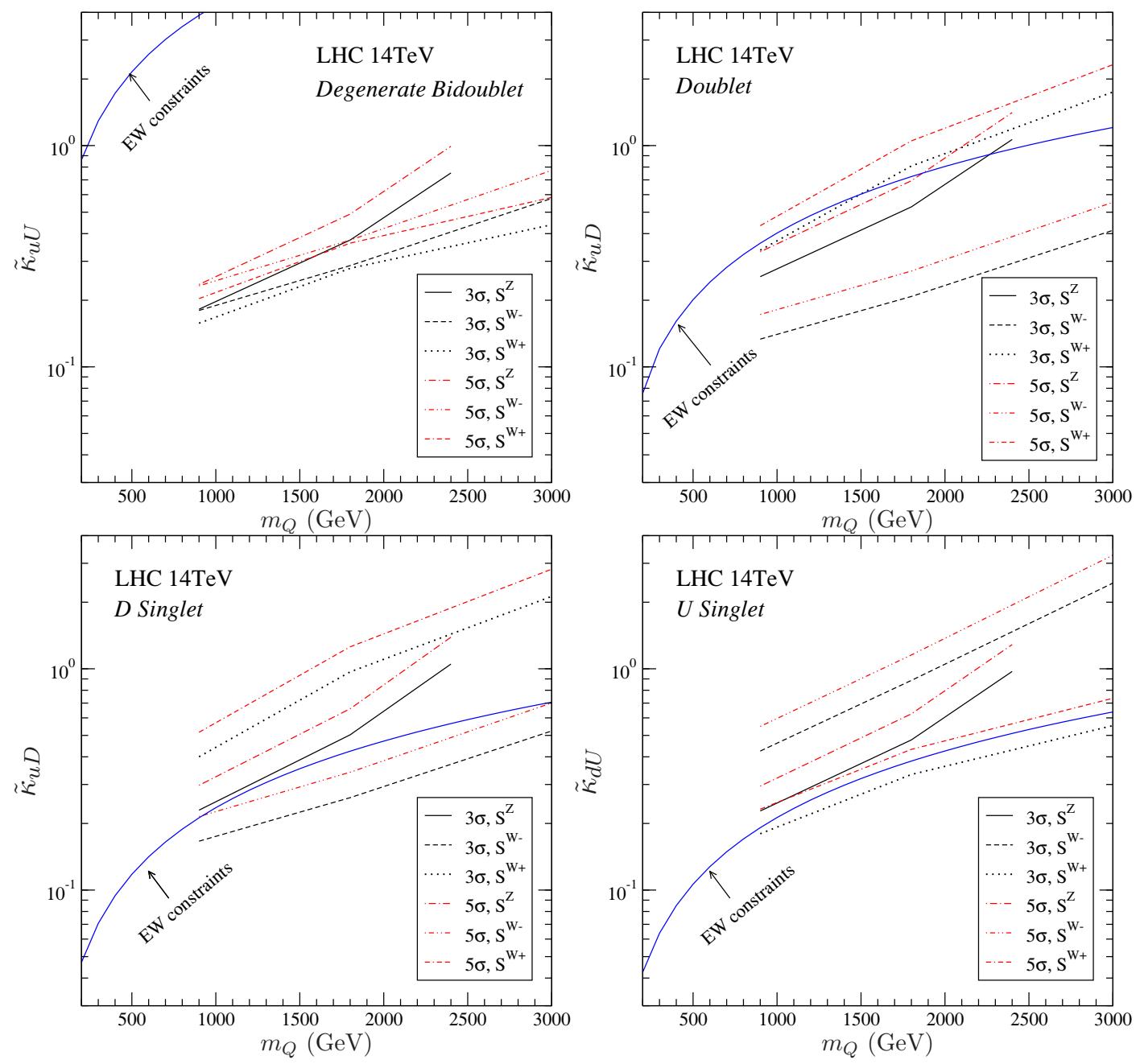

Figure 11. Same as figure 10 but for $\sqrt{s}=14 \mathrm{TeV}$ and $100 \mathrm{fb}^{-1}$ of data.

which is therefore smaller than $1 / 2$, and Yukawa couplings given in the notation of appendix B

$$
Y_{d D}=s_{L} c_{L} \frac{m_{D}}{v}, \quad Y_{D d}=s_{L} c_{L} \frac{m_{d}}{v} .
$$

These Yukawa couplings are needed to compute the corresponding branching fractions.

\subsection{2 $U$ type singlet}

A very similar model consists of an extra singlet $U$ with hypercharge $2 / 3$ and we assume that it mixes only with the first generation (doublet) in the basis in which all SM flavour occurs in the down quark sector. The model can be fully parameterized in terms of the physical up quark mass, $m_{u}$, the physical heavy up quark mass, $m_{U}$, and the coupling between $U$ and $d, \kappa_{d U}^{L} \equiv V_{u d} s_{L}$, with $V$ the CKM matrix and $s_{L}$ is used to denote the sine of an angle (and is therefore smaller than one). The decoupling limit occurs for $m_{U} \rightarrow \infty$, $s_{L} \rightarrow 0$ with $s_{L} m_{U}$ constant. In that limit all effects go to zero. The non-zero couplings 
apart from $\kappa_{d U}^{L}$, are

$$
\kappa_{u U}^{L}=s_{L} c_{L},
$$

which is therefore smaller than $1 / 2$, and Yukawa couplings given in the notation of appendix B

$$
Y_{u U}=s_{L} c_{L} \frac{m_{U}}{v}, \quad Y_{U u}=s_{L} c_{L} \frac{m_{u}}{v} .
$$

These Yukawa couplings are needed to compute the corresponding branching fractions.

\subsubsection{Doublet with hypercharge $1 / 6$}

We now consider one hypercharge $1 / 6$ doublet that only mixes with $u_{R}$ in the basis in which all SM flavor occurs in the down sector. Everything can be parameterized in terms of the up quark mass, $m_{u}$, the $D$ quark mass, $m_{D}$, and $\kappa_{u D}=-s_{R}$. The other non-vanishing couplings are

$$
\kappa_{u U}=-s_{R} c_{R}, \quad \kappa_{d U}^{L}=-\kappa_{u D}^{L}=s_{L},
$$

and a Yukawa coupling between $u$ and $U$ that we use to compute the branching fractions

$$
Y_{u U}=s_{R} c_{R} \frac{m_{u}}{v}, \quad Y_{U u}=s_{R} c_{R} \frac{m_{U}}{v},
$$

where the mass of the heavy $U$ quark is

$$
m_{U}=\frac{\sqrt{m_{D}^{2}-s_{R}^{2} m_{u}^{2}}}{c_{R}} .
$$

The two LH couplings are irrelevant in practice, since we have the relation

$$
s_{L}=\frac{s_{R} m_{u}}{m_{D}} \approx 0,
$$

for $m_{D}$ above current experimental limits.

\subsubsection{Degenerate bidoublet with hypercharge $1 / 6$ and $7 / 6$}

This model discussed in detail in ref. [4] and in section 2, consists of two new vector-like quark electroweak doublets, with hypercharges $1 / 6$ and $7 / 6$ respectively, that mix only with the up quark, in the basis of diagonal Yukawa couplings for the charge 2/3 SM quarks. The two doublets are degenerate, i.e. they have the same mass and Yukawa couplings to $u$ before EWSB, and the particle content of these two doublets is two charge $2 / 3$, one charge $-1 / 3$ and one charge $5 / 3$ quark. The three quarks of types $U, D$ and $X$ in our notation, have the following values of $\kappa$

$$
\kappa_{u U}=\sqrt{2} \kappa_{u D}=\sqrt{2} \kappa_{u X}=s_{R},
$$

where $s_{R}$ is the sine of the corresponding mixing angle which depends on the particular values of the model parameters. In this model, the generic bound from the unitarity of the mixing is saturated for $\kappa_{u U} \leq 1$ and is a bit more stringent for $\kappa_{u D}=\kappa_{u X} \leq 1 / \sqrt{2}$. Also the constraints from the oblique parameters impose a mild bound of $\kappa_{u U} \lesssim 0.75$ (assuming $\Delta S \leq 0.2)$ in the model under consideration.

In this model each of the quark decays with $100 \%$ branching ratio to the respective gauge boson, namely $\operatorname{Br}(U \rightarrow u Z)=\operatorname{Br}(D \rightarrow u W)=\operatorname{Br}(X \rightarrow u W)=100 \%$. 


\subsection{Systematic effects}

The analysis reported here gives only an estimate of the LHC reach. Although better results could be obtained if the signal selection was based on a Likelihood or Discriminant Variable technique, it must be noted that a number of systematic effects, which are difficult to estimate by simulation, have been neglected. In particular,

- No k-factors have been taken into account for the signals and backgrounds.

- Since the charge identification plays a significant role in the CC analysis and Delphes does not simulate charge misidentification, the likelihood of charge mismeasurement was studied at the generator level. As an example, $p_{T}$ and $\eta$ dependent parameterization of the ATLAS tracker response was taken from [50]. We find the misidentification fraction to be very small, only going up to $1.2 \%$ (0.9\%) for the electrons (muons) from the heaviest quark $(3 \mathrm{TeV})$ studied in this paper.

- Multijet events, which are abundant at the LHC, can fake the CC decay signal if jets are misreconstructed as leptons and if jet transverse energies are poorly measured, leading to a presence of missing energy in the events. These effects are very difficult to estimate by fast Monte Carlo simulation since they depend strongly on the criteria used for lepton identification and jet measurements. Nevertheless, we expect that with optimized experimental methods this source of background will be small. Indeed, for the D0 and CDF analyses [53, 54] it is almost negligible.

- The selection cuts have been optimized for specific mass values. In practice, a sliding window search would have these cuts re-optimized at intermediate mass points. In some cases, because of experimental uncertainties and to have more robust results, the cuts could be loosened. For example, the $p_{T}^{\text {sum }}$ cut on the tag jet plus heavy quark candidate system, as given in eq. (5.5), could be raised to $150 \mathrm{GeV}$ for the $14 \mathrm{TeV}$ analysis, at the cost of $\sim 10 \%$ loss in significance.

- At high luminosity, pileup effects can become very important. Pileup will affect the purity of the signal. The isolation efficiency and resolution of leptons will deteriorate and fake forward jets would be generated.

\section{Summary}

The Fermilab Tevatron has been leading the way for searches for new heavy particles, and the historical mission of the LHC at the energy and luminosity frontier will take the field of high energy physics to a new era. Among many highly motivated new particles in the $\mathrm{TeV}$ region, are new vector-like quarks that can have sizable couplings to first generation quarks without conflicting with current experimental constraints. The coupling with valence quarks and unique kinematics make single production at hadron colliders the optimal discovery process, potentially superior to the pair production mechanism via QCD interactions. 
In this article, we first motivated the consideration for the new heavy quarks by presenting a few theoretical models. We then discussed the existing experimental bounds on the quark mass and their couplings to the SM fields. In order to make our study as widely applicable as possible, we have performed a model independent-analysis of the discovery reach at the Large Hadron Collider for new vector-like quarks considering single production and subsequent decays via electroweak interactions. After an exploration of the unique features of the signal kinematics, we performed an exhaustive optimization for signal extraction over the SM backgrounds. With this optimized analysis, we determined the reach for the signal as a function of the heavy quark mass. The result is given in terms of model independent quantities that can be identified with production cross section times branching ratio of the heavy quark decay to the corresponding electroweak gauge boson and a SM quark. We then translated these model independent bounds into bounds on the parameters of four popular models, with two degenerate doublets of hypercharges $1 / 6$ and $7 / 6$; one single doublet with hypercharge $1 / 6$; one down type singlet and one up type singlet.

We considered two scenarios, one with a short LHC run with $1 \mathrm{fb}^{-1}$ integrated luminosity at $\sqrt{s}=7 \mathrm{TeV}$ and a longer run with $100 \mathrm{fb}^{-1}$ at $\sqrt{s}=14 \mathrm{TeV}$. The reach of the former has been found to be comparable, although typically slightly better, to the final Tevatron reach with $10 \mathrm{fb}^{-1}$ integrated luminosity. It is only in the case of the degenerate doublets the probed region by the Tevatron and the short LHC run are unconstrained by precision data. However, the reach dramatically improves both in terms of mass and couplings for the longer run, probing in all four models considerable regions of parameter space not constrained by electroweak precision observables. In particular, masses up to $\sim 3.7 \mathrm{TeV}$ can be probed with couplings of order one or equivalently, production cross sections times branching ratios as small as $2.6 \mathrm{fb}$. It is evident from these results that the discovery potential for new heavy quarks at the LHC is very encouraging.

\section{Acknowledgments}

We acknowledge interesting discussions with R. Sekhar Chivukula, Joseph Lykken, M. Rodriguez-Chala and Elizabeth Simmons. Fermilab is operated by Fermi Research Alliance, LLC under Contract No. DE-AC02-07CH11359 with the United States Department of Energy. AA was supported in part by the US National Science Foundation under grant PHY-0854889. The work of TH is supported in part by the United States Department of Energy under grant DE-FG02-95ER40896. JS is partially supported by projects FPA201017915, FPA2006-05294, FQM 101, FQM 03048 and by MICINN through a Ramón y Cajal contract and would like to thank CERN TH division for hospitality during completion of this work.

\section{A Explicit calculation of $\mathrm{S}$}

The one loop contribution to the $\mathrm{S}$ parameter from an arbitrary number of fermions is given by [9]

$$
S=\frac{3}{4 \pi} \sum_{i, j}\left[\left(U_{i j}^{L} Y_{j i}^{L}+U_{i j}^{R} Y_{j i}^{R}\right) \bar{\chi}_{+}\left(\mathcal{M}_{i i}, \mathcal{M}_{j j}\right)+\left(U_{i j}^{L} Y_{j i}^{R}+U_{i j}^{R} Y_{j i}^{L}\right) \bar{\chi}_{-}\left(\mathcal{M}_{i i}, \mathcal{M}_{j j}\right)\right]
$$


where the sum runs over all fermions, $\mathcal{M}_{i j}$ is the physical (diagonal) mass matrix, $U_{i j}^{L, R}$ are the couplings of left and right handed fermions to $W_{\mu}^{3}$, respectively and $Y_{i j}^{L, R}$ the corresponding couplings to $B_{\mu}$, all in the physical basis. We have also defined the following functions

$$
\begin{aligned}
\bar{\chi}_{+}\left(y_{1}, y_{2}\right)= & \frac{5\left(y_{1}^{4}+y_{2}^{4}\right)-22 y_{1}^{2} y_{2}^{2}}{9\left(y_{1}^{2}-y_{2}^{2}\right)^{2}}+\frac{2 y_{1}^{2} y_{2}^{2}\left(y_{1}^{2}+y_{2}^{2}\right)-y_{1}^{6}-y_{2}^{6}}{3\left(y_{1}^{2}-y_{2}^{2}\right)^{3}} \log \left(\frac{y_{1}^{2}}{y_{2}^{2}}\right) \\
& -\frac{1}{3}\left[\log \left(\frac{y_{1}^{2}}{\mu^{2}}\right)+\log \left(\frac{y_{2}^{2}}{\mu^{2}}\right)\right], \\
\bar{\chi}_{-}\left(y_{1}, y_{2}\right)= & \frac{y_{1} y_{2}}{\left(y_{1}^{2}-y_{2}^{2}\right)^{3}}\left[y_{1}^{4}-y_{2}^{4}-2 y_{1}^{2} y_{2}^{2} \log \left(\frac{y_{1}^{2}}{y_{2}^{2}}\right)\right] .
\end{aligned}
$$

The model with two exactly degenerate doublets that only mix with the up quark can be parameterized by three parameters which are usually taken to be the up Yukawa coupling, the Yukawa coupling between the up and the two doublets and the (common) mass of the two doublets. All these parameters are defined in the current eigenstate bases, i.e. before diagonalizing the mass matrix. It is however much more advantageous to define physical parameters which, in our case can be taken to be the (physical) mass of the up quark $m_{u}$, the physical mass of the three degenerate heavy states (which coincides with $M)$ and the coupling $\kappa_{u U}=s_{R}$, which coincides with the sine of the RH rotation needed to diagonalize the mass matrix. Full details of this parameterization can be found in ref. [21]. The physical masses, written in terms of these parameters and in a basis with first the (three) charge $2 / 3$ quarks, then the two charge $-1 / 3$ quarks and finally the charge $-5 / 3$ one are,

$$
\mathcal{M}=\operatorname{diag}\left(m_{u}, M, \frac{\sqrt{M^{2}-m_{u}^{2} s_{R}^{2}}}{c_{R}}, m_{d}, M, M\right),
$$

whereas the couplings relevant for the calculation of the $S$ parameter are, in the same basis

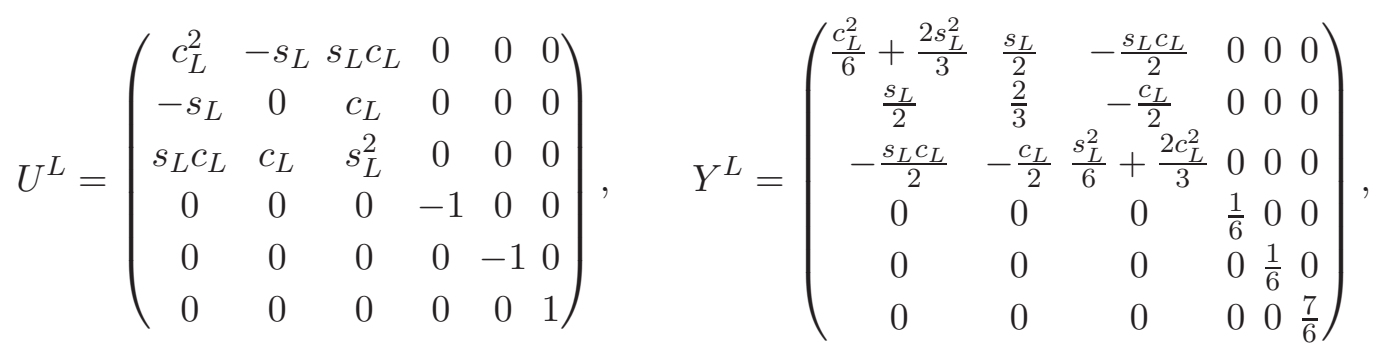

and

$$
U^{R}=\left(\begin{array}{cccccc}
0 & -s_{R} & 0 & 0 & 0 & 0 \\
-s_{R} & 0 & c_{R} & 0 & 0 & 0 \\
0 & c_{R} & 0 & 0 & 0 & 0 \\
0 & 0 & 0 & 0 & 0 & 0 \\
0 & 0 & 0 & 0 & -1 & 0 \\
0 & 0 & 0 & 0 & 0 & 1
\end{array}\right), \quad Y^{R}=\left(\begin{array}{cccccc}
\frac{2}{3} & \frac{s_{R}}{2} & 0 & 0 & 0 & 0 \\
\frac{s_{R}}{2} & \frac{2}{3} & -\frac{c_{R}}{2} & 0 & 0 & 0 \\
0 & -\frac{c_{R}}{2} & \frac{2}{3} & 0 & 0 & 0 \\
0 & 0 & 0 & -\frac{1}{3} & 0 & 0 \\
0 & 0 & 0 & 0 & \frac{1}{6} & 0 \\
0 & 0 & 0 & 0 & 0 & \frac{7}{6}
\end{array}\right)
$$

where the LH rotation is given in terms of our input parameters by $s_{L}=s_{R} m_{u} / M$ (see [21]). Eq. (A.6) shows that we indeed have $\kappa_{u U}=s_{R}$. We can now insert the explicit form of 
the couplings and masses in eq. (A.1) and expand in the small ratio $m_{u} / M \lesssim 10^{-6}$. The resut is

$$
S=S_{\mathrm{SM}}-\frac{6 s_{R}^{2}-9 s_{R}^{4}+5 s_{R}^{6}+3\left(2-4 s_{R}^{2}+3 s_{R}^{4}\right) \log \left[1-s_{R}^{2}\right]}{3 \pi s_{R}^{6}}+\mathcal{O}\left(\frac{m_{u}^{2}}{M^{2}}\right),
$$

where

$$
S_{\mathrm{SM}}=\frac{3-\log \left(m_{u}^{2} / m_{d}^{2}\right)}{6 \pi},
$$

is the SM contribution from the up and down quarks that we have to subtract to compute the new physics contribution to the $\mathrm{S}$ parameter. Thus, we see that, up to tiny corrections $\mathcal{O}\left(m_{u}^{2} / M^{2}\right) \lesssim 10^{-12}$, the new physics contribution to $\mathrm{S}$ in this model is independent of $M$ for fixed values of $\kappa_{u U}$.

\section{B Heavy fermion decay widths}

In this appendix we collect the general expressions for the decay widths needed in the calculation of the branching ratios for arbitrary models. The decay width for a two body decay of a heavy fermion, $Q$ with mass $m_{Q}$, into a light fermion, $q$ with mass $m_{q}$ and a massive gauge boson, $V$ with mass $m_{V}$, and couplings

$$
V_{\mu}\left[\bar{q} \gamma^{\mu}\left(g_{L} \mathcal{P}_{L}+g_{R} \mathcal{P}_{R}\right) Q+\text { h.c. }\right]
$$

reads

$$
\begin{aligned}
\Gamma(Q \rightarrow V q)= & \frac{\sqrt{m_{q}^{4}-2 m_{q}^{2}\left(m_{Q}^{2}+m_{V}^{2}\right)+\left(m_{Q}^{2}-m_{V}^{2}\right)^{2}}}{32 \pi m_{Q}^{3} m_{V}^{2}} \\
& \times\left\{\left(\left|g_{L}\right|^{2}+\left|g_{R}\right|^{2}\right)\left[\left(m_{Q}^{2}-m_{q}^{2}\right)^{2}+m_{V}^{2}\left(m_{Q}^{2}+m_{q}^{2}\right)-2 m_{V}^{4}\right]\right. \\
& \left.\quad-6\left(g_{L} g_{R}^{*}+g_{L}^{*} g_{R}\right) m_{Q} m_{q} m_{V}^{2}\right\} \\
\approx & \frac{\left(g_{L}^{2}+g_{R}^{2}\right)}{32 \pi} \frac{m_{Q}^{3}}{m_{V}^{2}}\left[1-3 \frac{m_{V}^{4}}{m_{Q}^{4}}+2 \frac{m_{V}^{6}}{m_{Q}^{6}}\right], \text { for }\left(m_{Q}, m_{V} \gg m_{q}\right),
\end{aligned}
$$

Similarly the decay into a quark and a scalar, with couplings,

$$
\frac{1}{\sqrt{2}} H\left[Y_{Q q} \bar{Q}_{L} q_{R}+Y_{q Q} \bar{q}_{L} Q_{R}+\text { h.c. }\right]
$$

is given by

$$
\begin{aligned}
\Gamma(Q \rightarrow H q)= & \frac{\sqrt{m_{H}^{4}+\left(m_{Q}^{2}-m_{q}^{2}\right)^{2}-2 m_{H}^{2}\left(m_{Q}^{2}+m_{q}^{2}\right)}}{64 \pi m_{Q}^{3}} \\
& \times\left[\left(Y_{Q q}^{2}+Y_{q Q}^{2}\right)\left[m_{Q}^{2}+m_{q}^{2}-m_{H}^{2}\right]+2\left(Y_{Q q} Y_{q Q}^{*}+Y_{Q q}^{*} Y_{q Q}\right) m_{Q} m_{q}\right] \\
\approx & \frac{Y_{Q q}^{2}+Y_{q Q}^{2}}{64 \pi} m_{Q}\left[1-2 \frac{m_{H}^{2}}{m_{Q}^{2}}+\frac{m_{H}^{4}}{m_{Q}^{4}}\right], \text { for }\left(m_{Q}, m_{H} \gg m_{q}\right) .
\end{aligned}
$$


Open Access. This article is distributed under the terms of the Creative Commons Attribution Noncommercial License which permits any noncommercial use, distribution, and reproduction in any medium, provided the original author(s) and source are credited.

\section{References}

[1] F. del Aguila, M. Pérez-Victoria and J. Santiago, Effective description of quark mixing, Phys. Lett. B 492 (2000) 98 [hep-ph/0007160] [SPIRES].

[2] F. del Aguila, M. Pérez-Victoria and J. Santiago, Observable contributions of new exotic quarks to quark mixing, JHEP 09 (2000) 011 [hep-ph/0007316] [SPIRES].

[3] F. del Aguila, J. de Blas and M. Pérez-Victoria, Effects of new leptons in electroweak precision data, Phys. Rev. D 78 (2008) 013010 [arXiv:0803.4008] [SPIRES].

[4] A. Atre, M. Carena, T. Han and J. Santiago, Heavy quarks above the top at the Tevatron, Phys. Rev. D 79 (2009) 054018 [arXiv:0806.3966] [SPIRES].

[5] K. Agashe, R. Contino, L. Da Rold and A. Pomarol, A custodial symmetry for Z $b \bar{b}$, Phys. Lett. B 641 (2006) 62 [hep-ph/0605341] [SPIRES].

[6] M.S. Carena, E. Ponton, J. Santiago and C.E.M. Wagner, Light Kaluza-Klein states in Randall-Sundrum models with custodial SU(2), Nucl. Phys. B 759 (2006) 202 [hep-ph/0607106] [SPIRES].

[7] G. Cacciapaglia, C. Csáki, G. Marandella and J. Terning, A new custodian for a realistic Higgsless model, Phys. Rev. D 75 (2007) 015003 [hep-ph/0607146] [SPIRES].

[8] R. Contino, L. Da Rold and A. Pomarol, Light custodians in natural composite Higgs models, Phys. Rev. D 75 (2007) 055014 [hep-ph/0612048] [SPIRES].

[9] M.S. Carena, E. Ponton, J. Santiago and C.E.M. Wagner, Electroweak constraints on warped models with custodial symmetry, Phys. Rev. D 76 (2007) 035006 [hep-ph/0701055] [SPIRES].

[10] M. Carena, A.D. Medina, N.R. Shah and C.E.M. Wagner, Gauge-Higgs unification, neutrino masses and dark matter in warped extra dimensions, Phys. Rev. D 79 (2009) 096010 [arXiv:0901.0609] [SPIRES].

[11] M.E. Albrecht, M. Blanke, A.J. Buras, B. Duling and K. Gemmler, Electroweak and flavour structure of a warped extra dimension with custodial protection, JHEP 09 (2009) 064 [arXiv: 0903.2415] [SPIRES].

[12] F. del Aguila, A. Carmona and J. Santiago, Neutrino masses from an $A_{4}$ symmetry in holographic composite Higgs models, JHEP 08 (2010) 127 [arXiv:1001.5151] [SPIRES].

[13] S. Casagrande, F. Goertz, U. Haisch, M. Neubert and T. Pfoh, The custodial Randall-Sundrum model: from precision tests to Higgs physics, JHEP 09 (2010) 014 [arXiv: 1005.4315] [SPIRES].

[14] M. Carena, A.D. Medina, B. Panes, N.R. Shah and C.E.M. Wagner, Collider phenomenology of gauge-Higgs unification scenarios in warped extra dimensions, Phys. Rev. D 77 (2008) 076003 [arXiv:0712.0095] [SPIRES].

[15] R. Contino and G. Servant, Discovering the top partners at the LHC using same-sign dilepton final states, JHEP 06 (2008) 026 [arXiv:0801.1679] [SPIRES]. 
[16] J.A. Aguilar-Saavedra, Identifying top partners at LHC, JHEP 11 (2009) 030 [arXiv:0907.3155] [SPIRES].

[17] J. Mrazek and A. Wulzer, A strong sector at the LHC: top partners in same-sign dileptons, Phys. Rev. D 81 (2010) 075006 [arXiv:0909.3977] [SPIRES].

[18] G. Dissertori, E. Furlan, F. Moortgat and P. Nef, Discovery potential of top-partners in a realistic composite Higgs model with early LHC data, JHEP 09 (2010) 019 [arXiv: 1005.4414] [SPIRES].

[19] D.E. Morrissey and C.E.M. Wagner, Beautiful mirrors, unification of couplings and collider phenomenology, Phys. Rev. D 69 (2004) 053001 [hep-ph/0308001] [SPIRES].

[20] K. Kumar, W. Shepherd, T.M.P. Tait and R. Vega-Morales, Beautiful mirrors at the LHC, JHEP 08 (2010) 052 [arXiv: 1004.4895] [SPIRES].

[21] F. del Aguila, A. Carmona and J. Santiago, Tau custodian searches at the LHC, Phys. Lett. B 695 (2011) 449 [arXiv: 1007.4206] [SPIRES].

[22] F. Gursey and M. Serdaroglu, Basic fermion masses and mixings in the $E_{6}$ model, Lett. Nuovo Cim. 21 (1978) 28 [SPIRES].

[23] F. Gursey, P. Ramond and P. Sikivie, A universal gauge theory model based on $E_{6}$, Phys. Lett. B 60 (1976) 177 [SPIRES].

[24] S. Sultansoy and G. Unel, The $E_{6}$ inspired isosinglet quark and the Higgs boson, Phys. Lett. B 669 (2008) 39 [hep-ex/0610064] [SPIRES].

[25] R. Mehdiyev, A. Siodmok, S. Sultansoy and G. Unel, Down type isosinglet quarks in ATLAS, Eur. Phys. J. C 54 (2008) 507 [arXiv:0711.1116] [SPIRES].

[26] F. del Aguila et al., Collider aspects of flavour physics at high Q, Eur. Phys. J. C 57 (2008) 183 [arXiv:0801.1800] [SPIRES].

[27] P.H. Frampton, P.Q. Hung and M. Sher, Quarks and leptons beyond the third generation, Phys. Rept. 330 (2000) 263 [hep-ph/9903387] [SPIRES].

[28] B. Holdom et al., Four statements about the fourth generation, PMC Phys. A 3 (2009) 4 [arXiv: 0904.4698] [SPIRES].

[29] G.D. Kribs, T. Plehn, M. Spannowsky and T.M.P. Tait, Four generations and Higgs physics, Phys. Rev. D 76 (2007) 075016 [arXiv:0706.3718] [SPIRES].

[30] J. Erler and P. Langacker, Precision constraints on extra fermion generations, Phys. Rev. Lett. 105 (2010) 031801 [arXiv:1003.3211] [SPIRES].

[31] H. Martinez, A. Melfo, F. Nesti and G. Senjanović, Three extra mirror or sequential families: a case for heavy Higgs and inert doublet, Phys. Rev. Lett. 106 (2011) 191802 [arXiv: 1101.3796] [SPIRES].

[32] O. Eberhardt, A. Lenz and J. Rohrwild, Less space for a new family of fermions, Phys. Rev. D 82 (2010) 095006 [arXiv: 1005.3505] [SPIRES].

[33] I. Gogoladze, B. He and Q. Shafi, New fermions at the LHC and mass of the Higgs boson, Phys. Lett. B 690 (2010) 495 [arXiv: 1004.4217] [SPIRES].

[34] F. del Aguila and M.J. Bowick, The possibility of new fermions with $\Delta I=0$ mass, Nucl. Phys. B 224 (1983) 107.

[35] M.E. Peskin and T. Takeuchi, Estimation of oblique electroweak corrections, Phys. Rev. D 46 (1992) 381 [SPIRES]. 
[36] F. del Aguila and M.J. Bowick, Suppression of lepton number violation mediated by $\Delta I=0$ mass fermions, Phys. Lett. B 119 (1982) 144 [SPIRES].

[37] Z. Han and W. Skiba, Effective theory analysis of precision electroweak data, Phys. Rev. D 71 (2005) 075009 [hep-ph/0412166] [SPIRES].

[38] Z. Han, Electroweak constraints on effective theories with $\mathrm{U}(2) \times \mathrm{U}(1)$ flavor symmetry, Phys. Rev. D 73 (2006) 015005 [hep-ph/0510125] [SPIRES].

[39] G.H. Brooijmans et al., New physics at the LHC: a Les Houches report. Physics at TeV Colliders 2007 - New Physics Working Group, arXiv:0802.3715 [SPIRES].

[40] G. Cacciapaglia, A. Deandrea, D. Harada and Y. Okada, Bounds and decays of new heavy vector-like top partners, JHEP 11 (2010) 159 [arXiv:1007.2933] [SPIRES].

[41] J. Pumplin et al., New generation of parton distributions with uncertainties from global QCD analysis, JHEP 07 (2002) 012 [hep-ph/0201195] [SPIRES].

[42] J. Alwall et al., MadGraph/MadEvent v4: the new web generation, JHEP 09 (2007) 028 [arXiv:0706.2334] [SPIRES].

[43] T. Sjöstrand, S. Mrenna and P.Z. Skands, PYTHIA 6.4 physics and manual, JHEP 05 (2006) 026 [hep-ph/0603175] [SPIRES].

[44] S. Ovyn, X. Rouby and V. Lemaitre, Delphes, a framework for fast simulation of a generic collider experiment, arXiv:0903.2225 [SPIRES].

[45] T. Han, G. Valencia and S. Willenbrock, Structure function approach to vector boson scattering in pp collisions, Phys. Rev. Lett. 69 (1992) 3274 [hep-ph/9206246] [SPIRES].

[46] T. Figy, C. Oleari and D. Zeppenfeld, QCD corrections to vector boson fusion processes, Nucl. Phys. Proc. Suppl. 135 (2004) 9 [hep-ph/0407066] [SPIRES].

[47] J. Alwall et al., Comparative study of various algorithms for the merging of parton showers and matrix elements in hadronic collisions, Eur. Phys. J. C 53 (2008) 473 [arXiv:0706.2569] [SPIRES].

[48] J. Alwall et al., New developments in MadGraph/MadEvent, AIP Conf. Proc. 1078 (2009) 84 [arXiv:0809.2410] [SPIRES].

[49] J.E. Gaiser, Charmonium spectroscopy from radiative decays of the $J / \Psi$ and $\Psi^{\prime}$, Ph.D. Thesis, Stanford University, Stanford U.S.A. (1982), SLAC-R-255 [SPIRES].

[50] ATLAS collaboration, G. Aad et al., Expected performance of the ATLAS experiment: detector, trigger and physics, CERN-OPEN-2008-020 [arXiv:0901.0512] [SPIRES].

[51] THE CDF collaboration, Search for heavy top $t^{\prime} \rightarrow W q$ in lepton plus jets events in $\int \mathcal{L} d t=4.6 \mathrm{fb}^{-1}$, CDF-Note-10110.

[52] The CDF collaboration, T. Aaltonen et al., Search for new particles leading to Z+jets final states in pp collisions at $\sqrt{s}=1.96$ TeV, Phys. Rev. D 76 (2007) 072006 [arXiv:0706.3264] [SPIRES].

[53] ThE CDF collaboration, Search for single production of heavy quarks, CDF-Note-10261.

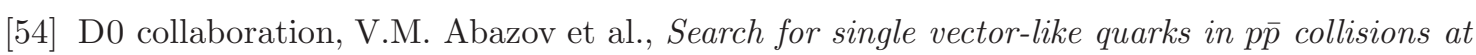
$\sqrt{s}=1.96 \mathrm{TeV}$, Phys. Rev. Lett. 106 (2011) 081801 [arXiv:1010.1466] [SPIRES].

[55] CMS collaboration, G.L. Bayatian et al., CMS physics: technical design report, CERN-LHCC-2006-001 [SPIRES]. 